\title{
$\beta$-adrenergic control of sarcolemmal Cav1.2 abundance by small GTPase Rab proteins
}

Silvia G. del Villar, Taylor L. Voelker, Heather C. Spooner, Eamonn J. Dickson, and Rose E. Dixon*.

Dept. of Physiology and Membrane Biology, University of California, Davis, CA

*To whom correspondence should be addressed:

Rose E. Dixon

University of California, Davis

Department of Physiology \& Membrane Biology

School of Medicine, Tupper Hall, room 4112

One Shields Avenue

Davis, California 95616

Phone: (530) 754-0742

Email: redickson@ucdavis.edu

https://orcid.org/0000-0002-5612-4761

https://orcid.org/0000-0002-6779-9350

https://orcid.org/0000-0002-0873-8021

https://orcid.org/0000-0001-8619-7630

https://orcid.org/0000-0003-0655-690X

\section{Classification}

BIOLOGICAL SCIENCES I Physiology

\section{Keywords}

L-type calcium channel I heart I $\beta$-adrenergic receptor I trafficking I clustering

\section{Author Contributions}

R.E.D. conceived experiments, R.E.D. and S.G.V. designed experiments, S.G.V., T.V., and H.C.S. executed experiments, S.G.V., T.V. and R.E.D. analyzed data and prepared figures, S.G.V. and R.E.D. wrote the paper, R.E.D. and E.J.D. interpreted data and all authors read and revised the manuscript.

\section{This PDF file includes:}

Main Text

Figures 1 to 7

Tables 1

SI Appendix 


\begin{abstract}
The number and activity of $\mathrm{Ca}_{v} 1.2$ channels in the cardiomyocyte sarcolemma tunes the magnitude of $\mathrm{Ca}^{2+}$-induced $\mathrm{Ca}^{2+}$ release and myocardial contraction. $\beta$-adrenergic receptor $(\beta A R)$ activation stimulates sarcolemmal insertion of Cav1.2 channels. This supplements the pre-existing sarcolemmal Cav1.2 population, forming large 'superclusters' wherein neighboring channels undergo enhanced cooperative-gating behavior, amplifying $\mathrm{Ca}^{2+}$ influx and myocardial contractility. Here, we determine this stimulated insertion is fueled by an internal reserve of early- and recycling endosome-localized, presynthesized Cav1.2 channels. $\beta A R$-activation decreased Cav1.2/endosome colocalization in ventricular myocytes, as it triggered 'emptying' of endosomal Cav1.2 cargo into the sarcolemma. We examined the rapid dynamics of this stimulated insertion process with live-myocyte imaging of channel trafficking, and discovered that Cav1.2 are often inserted into the sarcolemma as pre-formed, multi-channel clusters. Likewise, entire clusters were removed from the sarcolemma during endocytosis, while in other cases, a more incremental process suggested removal of individual channels. The amplitude of the stimulated insertion response was doubled by co-expression of constitutively-active Rab4a, halved by co-expression of dominant-negative Rab11a, and abolished by coexpression of dominant-negative mutant Rab4a. In ventricular myocytes, $\beta A R$-stimulated recycling of Cav1.2 was diminished by both nocodazole and latrunculin-A, suggesting an essential role of the cytoskeleton in this process. Functionally, cytoskeletal disruptors prevented $\beta A R$-activated $\mathrm{Ca}^{2+}$ current augmentation. Moreover, $\beta A R$-regulation of $\mathrm{Cav} 1.2$ was abolished when recycling was halted by co-application of nocodazole and latrunculinA. These findings reveal that $\beta A R$-stimulation triggers an on-demand boost in sarcolemmal Cav1.2 abundance via targeted, Rab4a and Rab11a-dependent insertion of Cav1.2 channels is essential for $\beta A R$-regulation of cardiac Cav1.2.
\end{abstract}




\section{Significance Statement}

The L-type voltage-gated $\mathrm{Ca}^{2+}$ channel $\mathrm{Cav1.2}$ is essential for excitation-contraction coupling in the heart. During the fight-or-flight response, Cav1.2 channel activity is augmented as a result of PKA-mediated phosphorylation, downstream of $\beta$-adrenergic receptor $(\beta A R)$ activation. We discovered that enhanced sarcolemmal abundance of Cav1.2 channels, driven by stimulated insertion/recycling of specific Cav1.2 containing endosomes, is essential for $\beta A R$-mediated regulation of these channels in the heart. These data reveal a new conceptual framework of this critical and robust pathway for ondemand tuning of cardiac EC-coupling during fight-or-flight. 


\section{Main Text}

\section{Introduction}

$\mathrm{Ca}^{2+}$ influx through L-type $\mathrm{Ca}^{2+}$ channels $(\mathrm{Cav} 1.2)$ is indispensable for cardiac excitationcontraction coupling (EC-coupling) (1). These multimeric proteins consist of a poreforming and voltage-sensing $a_{1 c}$ subunit, and auxiliary $\beta$ and $a_{2} \delta$-subunits. In ventricular myocytes, Cav1.2 mainly localize to the t-tubule sarcolemma and open briefly, allowing a small amount of $\mathrm{Ca}^{2+}$ influx, in response to the wave of depolarization that travels through the conduction system of the heart from its point of origin, usually in the SA-node. This initial influx is amplified manifold though $\mathrm{Ca}^{2+-i n d u c e d ~} \mathrm{Ca}^{2+}$ release $(\mathrm{CICR})$ from juxtaposed type 2 ryanodine receptors (RyR2) on the junctional sarcoplasmic reticulum (jSR), Which are localized a short $\sim 12 \mathrm{~nm}$ across the dyadic cleft. The synchronous opening of thousands of RyR2 generates a transient, global elevation in intracellular calcium concentration $\left(\left[\mathrm{Ca}^{2+}\right]_{\mathrm{i}}\right)$, resulting in contraction. Reducing Cav1.2 channel current $\left(I_{\mathrm{Ca}}\right)$ results in less $\mathrm{CICR}$, smaller $\left[\mathrm{Ca}^{2+}\right]_{i}$ transients, and less forceful contractions. Conversely, larger amplitude $I_{\mathrm{Ca}}$ elicits greater $\mathrm{Ca}^{2+}$ release from the $\mathrm{SR}$, producing more forceful contractions. The level of $\mathrm{Ca}^{2+}$ influx through Cav1.2 channels therefore tunes ECcoupling.

$I_{\mathrm{Ca}}$ is a product of the number of channels in the sarcolemma and their open probability $\left(P_{0}\right)$. Consequently, there are two possible, non-mutually exclusive strategies that may be adopted to alter $I_{\mathrm{Ca}}$ and consequently the magnitude of EC-coupling: i) adjust Cav1.2 channel activity $\left(P_{0}\right)$ and/or, ii) modify sarcolemmal Cav1.2 channel expression $(M)$. The first strategy of increasing channel $P_{\mathrm{o}}$ has long been associated with $\beta$-adrenergic receptor $(\beta A R)$-mediated signaling in the heart (2-4). During acute physical or emotional stress, norepinephrine spills from sympathetic varicosities onto cardiomyocytes, activating $\beta A R s$. The ensuing $G_{\text {as }}$ /adenylyl cyclase/cAMP/PKA signaling cascade culminates in PKA phosphorylation of several effector proteins, including Cav1.2 (or an element of their interactome $(5,6))$, enhancing their activity to generate this positive inotropic response.

As to the second strategy to increase $l_{\mathrm{Ca}}$, there remains a paucity of information regarding the mechanisms regulating Cav1.2 channel abundance in the cardiomyocyte sarcolemma. Classical secretory transport literature suggests that Cav1.2 channels are trafficked from 
the endoplasmic reticulum (ER) to the trans-Golgi-network (TGN) and onward to their dyadic position in the sarcolemma. Underscoring the importance of faithful Cav1.2 channel trafficking, altered Cav1.2 channel density has been reported in both failing $(7,8)$ and aging (9) ventricular myocytes, and impaired anterograde trafficking of Cav1.2 channels to the t-tubules of human ventricular myocytes has been linked to dilated cardiomyopathy (10). Yet, despite the importance of tight homeostatic control of Cav1.2 channel trafficking to prevent $\mathrm{Ca}^{2+}$ dysregulation, the molecular steps defining Cav1.2 channel sorting and insertion remain poorly understood. Therefore, elucidation of the trafficking pathways that regulate Cav1.2 channel abundance is critical for our understanding of the pathophysiology of heart failure and myocardial aging, and could potentially reveal new therapeutic or rejuvenation targets. Along that vein, in the treatment of cystic fibrosis, multiple drugs are in various stages of use or development to improve trafficking to, or to amplify, or stabilize, CFTR channels at the apical membrane of airway epithelial cells (11).

There have been no measurements of Cav1.2 channel lifetimes in cardiomyocytes, but pulse-chase experiments in immortalized cell lines support a lifetime of plasma membrane (PM)-localized Cav1.2 channels of $\sim 3 \mathrm{~h}$ (12) while total cellular Cav1.2 lifetime is $>20 \mathrm{~h}$ $(13,14)$. This disparity suggests membrane-Cav1.2 turns over much more dynamically than the total cellular channel content and implies ongoing local control by endosomal trafficking. Disturbance of the equilibrium between channel insertion/recycling and internalization would be predicted to lead to alterations in sarcolemmal Cav1.2 channel abundance. Trafficking of vesicular cargo through the endosomal pathway is regulated by Rab-GTPases, a >60-member family within the larger Ras superfamily of small GTPases (15-18). Rab5 is involved in endocytosis and control of vesicular cargo influx into early endosomes (EEs; also called sorting endosomes), while Rab4 controls efflux of cargo out of EEs and fast recycling ( $t_{1 / 2} \sim 1-2$ min) back to the PM (19). Rab11, expressed on recycling endosomes (RE; also called the endocytic recycling compartment or $E R C$ ), regulates slow recycling ( $\mathrm{t}_{1 / 2} \sim 12 \mathrm{~min}$ ) of cargo from this compartment back to the PM (19). In cortical neurons and pancreatic $\beta$-cells, activity-dependent Cav1.2 channel internalization has been postulated to play important roles in $\mathrm{Ca}^{2+}$ homeostasis, with implications for homeostatic synaptic plasticity and insulin production respectively (20). In mouse neonatal cardiomyocytes, Rab11b has been reported to limit expression PM Cav1.2 (21), while recent studies performed in HEK and HL-1 cells reported that endocytic 
recycling of cardiac Cav1.2 channels, regulates their surface abundance $(13,22)$. Despite this crucial information from other cell-types, there has been a lack of rigorous investigations, at the molecular level, into how Cav1.2 channel recycling is regulated in cardiac myocytes.

Here, we identify a dynamic, sub-sarcolemmal pool of Cav1.2-cargo-carrying endosomes that are rapidly mobilized to the ventricular myocyte sarcolemma along targeted Rab4a and Rab11a GTPase-regulated recycling pathways in response to $\beta A R$-stimulation. Using electrophysiology, cell biology approaches, TIRF and super-resolution microscopy, we report that enhanced sarcolemmal Cav1.2 abundance via targeted, ISO-stimulated, recycling of Cav1.2 channels is essential for $\beta A R$-regulation of cardiac Cav1.2. 


\section{Results}

\section{Internal pools of pre-synthesized Cav1.2 channels reside on endosomes}

Recently, we reported that sarcolemmal Cav1.2 channel expression increases in the heart during $\beta A R$ signaling (23). Activation of $\beta A R$ s with isoproterenol (ISO) produced a rapid, PKA-dependent augmentation of Cav1.2 channel abundance along ventricular myocyte ttubules. We hypothesized that an endosomal pool of Cav1.2 fuels the rapid, ISOstimulated insertion of channels into the sarcolemma of ventricular myocytes. To test this, we performed an examination of the distribution of Cav1.2 channels on EEs, REs and late endosomes (LEs) in adult mouse ventricular myocytes (AMVMs) using two- or three-color Airyscan super-resolution microscopy. Immuno-stained Cav1.2 channel cargo was observed on $15.1 \pm 0.4 \%$ of early endosome antigen-1 (EEA1) positive pixels in male ventricular myocytes and on a similar $16.1 \pm 0.7 \%$ in female myocytes (Figure $1 \mathrm{~A}$ and $\mathrm{B}$; $P=0.24$; see also Figure S1). Pre-fixation stimulation with $100 \mathrm{nM}$ ISO led to a significant, $15-20 \%$ decrease in colocalization between Cav1.2 and EEA1 (male $P=0.001$, female $P$ $=0.01$ ). We further narrowed our analysis to Rab4 positive EEs by performing triple-label experiments, co-staining for Cav1.2, EEA1, and Rab4 (Figure S2). A similar trend was observed there such that $15.6 \pm 1.3 \%$ of EEA1- and Rab4 co-expressing pixels were colocalized with Cav1.2, falling to $10.8 \pm 0.6 \%$ in ISO-stimulated cells $(P=0.008)$. These data suggest that ISO activation of $\beta A R \mathrm{~s}$, stimulates movement of $\mathrm{Cav} 1.2$ out of EEs and into another cellular compartment.

Cargo exiting the EE can be routed either to REs, LEs, or back to the sarcolemma via the fast recycling pathway (see working model in Figure $7 A$ ) $(17,19)$. If $\beta A R$ activation stimulates Cav1.2 channel trafficking from EEs into REs then a testable prediction is that ISO should increase colocalization between Cav1.2 and Rab11 (a marker of REs). Accordingly, we examined the distribution of Cav1.2 on Rab11-positive REs, and found a population of Cav1.2-cargo-carrying REs, where Cav1.2 colocalized with $14.9 \pm 0.5 \%$ of Rab11-positive pixels in males and with $13.3 \pm 0.6 \%$ in females (Figure 1C and D). An $18 \%$ decrease in colocalization between $\mathrm{Cav}_{\mathrm{v}} 1.2$ and Rab11 was observed in male cells treated with $100 \mathrm{nM}$ ISO $(P=0.002)$. Data from female cardiomyocytes showed a similar downward trend in colocalization. These results do not support the hypothesis that Cav1.2 channels move from Rab4-positive EEs into REs in response to ISO. We next tested the 
hypothesis that ISO stimulation drives the trafficking of Cav1.2 channels from EEs to LEs, however despite identifying a population of $\mathrm{Ca}_{v} 1.2$ channels on Rab7-positive LEs in male and female ventricular myocytes, ISO stimulation did not significantly alter the degree of Rab7/Cav1.2 colocalization (Figure 1E and F; $P=0.28$ in males, $P=0.39$ in females). Having ruled out two of the three possibilities, we surmise that $\beta A R$ stimulation drives an intracellular pool of Cav1.2 channels from Rab4-positive EEs into the fast recycling pathway, and Rab11-positive REs into the slow recycling pathway.

\section{ISO-stimulated enhancement of Cav1.2 recycling is regulated by Rab4 and Rab11}

Having determined that $\beta A R$ stimulation decreases the number of $\mathrm{Cav} 1.2$ channels on EEs and REs we next tested if Rab4-dependent fast and, Rab11-dependent slow recycling pathways facilitate enhancement of Cav1.2 delivery into the PM of transiently transfected tsA201 cells. While these cells do not recapitulate all of the intricacies of signaling in cardiomyocytes, we utilized them here because, 1) they provide a reductionist framework on which to test our Rab4 and Rab11 hypotheses in the absence of other voltage-gated channels; 2) they can be easily transfected, permitting manipulation of Rab-protein complement; and 3) they endogenously express $\beta A R s$ (24). PM expression of Cav1.2 channels was monitored during activation of $\beta A R s$ with $100 \mathrm{nM}$ ISO. Clusters of channels were readily identified in the TIRF footprint of the cell (Figure 2A). Upon wash-in of ISO, Cav1.2-tagRFP intensity in the TIRF footprint increased by an average of 1.37-fold over a period of minutes (Figure $2 \mathrm{~B} ; \tau_{\mathrm{on}}=2.26 \pm 0.02 \mathrm{~min}$ ), in close agreement with previous results showing a $44.9 \pm 6.2 \%$ increase in channels in ISO-stimulated AMVM sarcolemmas (23). An increased density (number/ $\mu \mathrm{m}^{2}$ ) of channel clusters in the TIRF footprint contributed to this elevation (Figure $2 \mathrm{C}$; $P<0.0001$ ). These results suggest that ISO stimulates enhanced recycling of Cav1.2 channels to the PM.

We tested the role of Rab4a in this dynamic response by co-expressing a mutant Rab4a with a single amino acid substitution (Q67L) that renders it resistant to GTP-hydrolysis, locking it in a GTP-bound, constitutively active state (CA-Rab4aQ67L; Figure 2D-F) (25). In these cells, addition of ISO stimulated a 1.69-fold increase in intensity, almost twice the maximal response observed in controls, and increased cluster density 2.4-fold (Figure 2F; $P=0.002$ ). Interestingly, co-expression of CA-Rab4a had no effect on basal Cav1.2 cluster density before addition of ISO ( $P=0.08$; Figure $2 \mathrm{C}$ and $\mathrm{F})$ indicating that even in this GTP- 
locked active state, ISO-stimulation is required to trigger the boost in Cav1.2 recycling. After ISO, the GTP-bound CA-Rab4a facilitates a larger, faster (1.76 $\pm 0.02 \mathrm{~min}$ ) recycling response. This suggests that Rab4a plays a role in $\beta A R$-stimulated channel recycling but also implies the involvement of an upstream effector.

To support this postulate, we examined the ISO response in cells expressing a dominantnegative, GDP-locked variant of Rab4 (DN-Rab4 ${ }^{\mathrm{S} 22 \mathrm{~N}}$ ). Under these conditions, ISOapplication failed to enhance Cav1.2 surface expression and instead a slight decrease in Cav1.2-tagRFP intensity and cluster density was observed in the PM over the course of the experiment (Figure 2G, H, and I). These results confirm that Rab4a is part of the essential trafficking machinery that underlies the ISO-stimulated enhanced recycling of Cav1.2.

Since a subpopulation of Cav1.2 channels that localize to Rab11 positive REs was also identified in AMVMs (Figure 1C and D), we tested the role of Rab11a in this dynamic recycling response to ISO using a dominant negative, GDP-locked DN-Rab11a ${ }^{\mathrm{S} 25 \mathrm{~N}}$. Despite impaired Rab11a function, an ISO-stimulated increase in Cav1.2-tagRFP intensity was still evident, albeit to a lesser extent than in controls (1.12-fold; Figure $2 \mathrm{~J}$ and $\mathrm{K}$ ). This was accompanied by a 1.49-fold increase in cluster density $(P=0.009$; Figure $2 \mathrm{~L})$. Accordingly, DN-Rab11a-mediated knock down of Rab11a activity generated $\sim 33 \%$ of the response seen in controls while knock down of Rab4a abolished the response (Figure $2 \mathrm{H}$ and $M)$. These data suggest that upstream Rab4a activity is necessary, not only for fast recycling to the PM but also for transfer of Cav1.2 cargo from EEs to REs. Impaired Rab4a function creates a 'road block' in the endosomal recycling system. The role of Rab4a in fast recycling was unmasked in cells with knocked down Rab11a activity, where $\tau_{\text {on }}$ of the stimulated recycling response was $0.96 \pm 0.02$ min (Figure $2 \mathrm{~K}$ ), significantly faster than controls where both Rab4a and Rab11a were active (2.26 $\pm 0.02 \mathrm{~min})$. A theoretical time course of the slow Rab11a-dependent contribution was calculated by subtracting the predominantly Rab4a-mediated recycling time-course in DN-Rab11a expressing cells from endogenous Rab controls (Figure 2M). This theoretical Rab11a-dependent response displayed a 1.29-fold increase in Cav1.2-tagRFP intensity and was well-fit with a single exponential function with a $\tau_{o n}=2.69 \pm 0.04$ min that was slower than control or Rab4amediated responses. Based on these data, our results suggest that Cav1.2 channels 
stimulated to recycle to the PM in response to $\beta A R$ activation are sourced approximately $1 / 3^{\text {rd }}$ from the fast Rab4a, and $2 / 3^{\text {rd }}$ from the slow Rab11a recycling pathways.

\section{Actin and microtubule disruption impairs ISO-stimulated Cav1.2 recycling}

We used Airyscan super-resolution microscopy to examine Cav1.2 channel proximity to microtubules (MTs) and actin filaments (Figure 3A-B). Immunostaining with a-tubulin revealed the extensive MT cytoskeleton with its lattice, grid-like appearance in the subsarcolemma, transitioning to a more longitudinally-oriented network deeper in the cell interior (Figure 3A). Cav1.2 channels decorate the MTs in both locations. Phalloidinstaining of the actin network revealed the periodic alignment of sarcomeric actin (Figure 3B). It is notoriously difficult to visualize cortical actin in cardiomyocytes due to the overwhelming abundance of sarcomeric actin but in many locations Cav1.2 channels were co-localized with actin (Figure 3B, white arrowheads). The degree of colocalization between Cav1.2 and, in particular MTs, implies a role for the cytoskeleton in regulating channel availability.

To study the role of these cytoskeletal highways in $\beta A R$-stimulated Cav1.2 mobilization from endosomes, we examined EEA1-localized Cav1.2 channel populations in AMVMs treated with cytoskeletal disruptors. Accordingly, freshly isolated myocytes were treated for 2 hrs with $10 \mu \mathrm{M}$ nocodazole, a drug known to prevent addition of tubulin to dynamic MTs, and depolymerize the stable variety (26). Immuno-staining with anti-a-tubulin confirmed the treatment had substantially disordered the MTs (Figure 3C). In this and upcoming experimental series, we refer to cells that did not receive cytoskeletal disruptors as 'untreated'. As in untreated cells (Figure 1A and B), Cav1.2 was observed to colocalize with a sub-population of EEs (16.9 $\pm 0.6 \%$; Figure 3D and E). However, in cells stimulated with $100 \mathrm{nM}$ ISO prior to fixation, the reduction in colocalization between EEA1 and Cav1.2 we had previously observed in untreated cells was absent in nocodazole-treated cells, instead remaining at $17.5 \pm 0.7 \%(P=0.51$ compared to nocodazole-treated control). These data suggest that MT network disruption prevents the 'emptying' of Cav1.2 channel cargo from the EEs into the sarcolemma and support a role for MTs and their associated motor proteins as conduits for ISO-stimulated Cav1.2 recycling. 
We then examined the role of the actin cytoskeleton by disrupting it with latrunculin A (latA; $5 \mu \mathrm{M}$ for $2 \mathrm{hrs}$ ), which both facilitates F-actin depolymerization (27) and prevents polymerization by sequestering actin monomers (26). Importantly, lat-A specifically affects the actin cytoskeleton but spares the MT network (28). Alexa Fluor 647-conjugated phalloidin staining of filamentous-actin (F-actin) was used to visually confirm lat-A mediated actin-disruption (Figure 3F). In lat-A-treated myocytes, Cav1.2 and EEA1 colocalization was similar to untreated controls $(16.2 \pm 0.7 \%$ versus $15.1 \pm 0.4 \% ; P=$ 0.14). However, ISO-stimulation did not affect colocalization levels $(16.7 \pm 0.5 \% ; P=0.64$; Figure $3 G$ and $H$ ). In addition, in cells co-treated with nocodazole and lat-A, ISOstimulation actually promoted a small, but significant increase in colocalization between Cav1.2 and EEA1 ( $P=0.03$; Figure $3 \mathrm{I}$ and $\mathrm{J})$. These data indicate a profound alteration in the endosomal pathway where recycling and or endocytosis have been impaired, creating an 'endosomal traffic jam' leading to accumulation of cargo on the endosomes, with no cytoskeletal highways to transport the cargo to its destination.

\section{Cytoskeletal disruption alters ISO-stimulated Cav1.2 dynamics and recycling}

Real-time visualization and quantification of the effects of cytoskeletal disruption on channel trafficking was performed using transduced AMVMs isolated from mice that had received a retro-orbital injection of $A A V 9-C a v \beta_{2 a}-p a G F P$. This auxiliary subunit of Cav1.2 binds to the pore-forming subunit with a 1:1 stoichiometry and acts in this context as a biosensor reporting the location of the subset of Cav1.2 $\mathrm{a}_{1 \mathrm{c}}$ it interacts with. This approach was previously validated by our group (23), with super-resolution microscopy experiments confirming that the biosensor and the $a_{1 c}$ colocalize, and unlike overexpression of $a_{1 c}$, at these concentrations we have found that $C_{a v} \beta_{2 a}$-paGFP transduction does not appreciably affect $\mathrm{Ca}_{v} 1.2 \mathrm{a}_{1 \mathrm{c}}$ expression as indicated by unaltered basal channel cluster sizes. A further advantage of this approach is that it requires no culturing of AMVMs which are known to rapidly dedifferentiate in culture (29). We began by examining the dynamic channel trafficking response to ISO in untreated AMVMs (i.e., in the absence of cytoskeletal disruptors) using TIRF microscopy. Discrete puncta of Cav $\beta_{2 a}$-paGFP decorated the TIRF footprint of the myocyte during control frames and additional puncta/clusters were seen to appear in the TIRF footprint supplementing the initial complement after perfusion with $100 \mathrm{nM}$ ISO (Movie 1 and Figure 4A). Given our 
endosome/Cav1.2 immunostaining results (Figure 1), this may represent endosomal cargo mobilized in response to ISO from subsarcolemmal locations deeper within the cell. In agreement with this, 3D-plots of Cav $\beta_{2 a}$-paGFP intensity over time and cell depth, constructed from 4D-spinning disk confocal experiments performed on transduced AMVMs at $37^{\circ} \mathrm{C}$, indicated $\mathrm{Cav} \beta_{2 a}$-paGFP was mobilized from several microns within the cell, and moved toward the surface in response to ISO (Figure S3). At physiological temperature, following a delay of $\sim 3 \mathrm{sec}$, the response to ISO proceeded monoexponentially with a $\tau=2.53 \pm 0.28 \mathrm{sec}$ until $\mathrm{Ca}_{v} \beta_{2 a}$-paGFP intensity reached $a$ plateau (Figure S3C-D), presumably achieved when the endosomal pool of channels had been depleted and balance between insertion and endocytosis reached a new equilibrium. These data fit well with previous measurements of the time course of $I_{\mathrm{Ca}}$ responses to ISO $(30,31)$. Interestingly, exchange of the perfusate with one containing $100 \mathrm{nM}$ of angiotensin II, quickly reversed the response, and a subset of $\mathrm{Ca}{ }_{v} \beta_{2 a}-p a G F P$ moved back into the interior of the cell with a $\tau=2.94 \pm 0.96 \mathrm{sec}$. Previous work performed at room temperature reported that prolonged (30 - $60 \mathrm{~min}$ ) application of angiotensin II produces Cav1.2 channel internalization (32), but here at physiological temperature, the time course was significantly accelerated.

To test the hypotheses that ISO treatment increases sarcolemmal expression of Cav1.2 by stimulating channel insertion/recycling, and that cytoskeletal highways carry these recycling channels to their destination, we performed 'image math' (see Methods) on TIRF time series to quantify the subpopulations of $\mathrm{Cav}_{2} \beta_{2 a}$-paGFP in the TIRF-footprint that were: i) inserted, ii) endocytosed, and iii) stably expressed during ISO stimulation. Responses to ISO in untreated AMVMs were compared, to those in cells treated with nocodazole, lat-A, or a combination of both (Figure 4). Live cell time series experiments revealed a dynamic population of $C a v \beta_{2 a}-p a G F P$ in all cells examined (see Movies 1-4), although the dynamics were appreciably less in cells that received cytoskeletal disrupting treatments, supporting the idea that both F-actin and MTs are important conduits of this response. The number of channels at the sarcolemma at any given time, is dictated by the balance between channel insertions via the biosynthetic delivery and endosomal recycling pathways, and channel removals via endocytosis. In untreated cells, ISO stimulation heavily shifted the balance in favor of insertion, implying a stimulated insertion/recycling 
process (Figure 4A, and $\mathrm{E}-\mathrm{G}$ ). This mismatch between insertion and endocytosis produced a $27.95 \pm 4.31 \%$ increase in sarcolemmal Cav $\beta_{2 a}$-paGFP expression (Figure $4 \mathrm{E})$. The time course of ISO-stimulated insertions of channels in untreated AMVMs can be observed in the ROls highlighted in Figure 4A. Examination of these time courses revealed rapid step-like insertion profiles, suggesting that Cav1.2 appear to often insert into the sarcolemma as preformed clusters, containing many channels (Figure 4A(i-iii), B(ii and iii), C(ii and iii), D(i and ii)). In some cases, a delivery hub was evident, where a succession of channel clusters appeared to insert one after the other (Figure 4A(ii) and $B(i i i))$. Channel endocytosis also appeared to involve removal of channel clusters in some instances (Figure 4C(i), D(iii)), while in other ROls, a slower, gradual removal of potentially individual channels was more evident (Figure 4B(i)).

Recycling of endosomal cargo back to the PM is known to rely on both MTs and actin (33). Indeed, cytoskeletal disruption reduced the magnitude of the ISO-stimulated augmentation of sarcolemmal $\mathrm{Ca}_{\mathrm{v}} \beta_{2 \mathrm{a}}$-paGFP expression compared to untreated cells (Figure 4E). Interestingly, how the two elements of the cytoskeleton affected the ISOstimulated change in sarcolemmal expression varied somewhat, as revealed by examination of insertion and endocytosis events in each AMVM cohort. Anterograde transport and targeting of Cav1.2 to the t-tubule membrane is known to occur along MTs, anchored there via the BAR-domain containing protein, BIN1 (A.K.A. amphiphysin II) (34). Here, our experiments were focused not on long distance trafficking from the trans-golgi to the membrane, but rather from the local endosome pool of channels and thus channel dynamics were observed over short 3 min periods before and after application of ISO. Our data indicates that nocodazole mediated MT disruption reduced ISO-stimulated insertion of Cav $\beta_{2 a}$-paGFP by an average of $\sim 70 \%$ compared to untreated cells (Figure $4 \mathrm{~F}$ ). Channel internalization and the lifetime of channels in the membrane was not affected by the degree of MT disruption tested here, as both endocytosed and static channel population sizes were not significantly altered by this treatment (Figure $4 \mathrm{G}$ and $\mathrm{H}$ ).

Actin disruption with lat-A in contrast had no significant effect on channel insertion compared to untreated cells (Figure 4F). In neurons and HEK293 cells, interactions between $C_{v} 1.2$ and the actin-interacting protein a-actinin have been found to stabilize Cav1.2 channel expression at the $\operatorname{PM}(35,36)$. A notable trend toward increased 
endocytosis of $\mathrm{Cav}_{\mathrm{v}} \beta_{2 \mathrm{a}}$-paGFP in lat-A treated cells was detected but this failed to reach significance assessed by a one-way ANOVA test (Figure 4G). There was also a trending increase in the fraction of stable Cav $\beta_{2 a}-$ paGFP in the TIRF footprint that appeared to be left 'stranded' there (Figure $4 \mathrm{H}$ ), perhaps reflective of incomplete internalization of Cav $\beta_{2 a}$ paGFP due to the lack of actin dynamics and the disrupted cortical actin network. This effect was not statistically different from controls but was significantly different from nocodazole treated cells. Finally, combined treatment with nocodazole and lat-A actually reduced the overall expression of Cav $\beta_{2 a}$-paGFP in the TIRF footprint by $8.63 \pm 6.76 \%$ over the course of the experiment (Figure 4F and G). This occurred when the balance of endocytosis and insertion/recycling shifted in favor of endocytosis. Collectively, these data suggest that MTs and actin are both important conduits of $\beta A R$-stimulated Cav1.2 recycling, with MTs playing the major role in channel insertion.

\section{BAR mediated Ica regulation is abrogated by cytoskeletal disruption}

To ascertain whether ISO-stimulated recycling of Cav1.2 channels into the cardiomyocyte sarcolemma makes any functional contribution to the $\beta A R$ regulation of these channels, we performed whole cell patch clamp recordings on freshly isolated AMVMs under various cytoskeletal disrupting conditions. Stimulation of untreated cardiomyocytes with $100 \mathrm{nM}$ ISO, produced a significant $1.65 \pm 0.19$-fold enhancement of $I_{\mathrm{Ca}}(P=0.04$; Figure $5 \mathrm{~A}$ and B) and caused a $13.33 \pm 1.49 \mathrm{mV}$ leftward-shift in the voltage-dependence of conductance (measured as the difference between $\mathrm{V}_{1 / 2}$ of each fit; $P<0.0001$; Figure $5 \mathrm{C}$ and Table 1 ). In addition, we observed an increase in the slope steepness of the Boltzmann function used to fit the $\mathrm{G} / \mathrm{G}_{\max }$ data from $5.45 \pm 0.84$ in controls to $4.90 \pm 1.06$ in $100 \mathrm{nM}$ ISO (Figure $5 \mathrm{C}$ and Table 1), suggesting a potential increase in cooperative gating behavior (23).

The impact of MT disruption on the $I_{\text {Ca }}$ response to ISO was tested in AMVMs incubated with nocodazole. This dose and duration of treatment did not alter control $I_{\mathrm{Ca}}$ amplitude (compared to untreated control). Indeed, none of the cytoskeletal disrupting treatments had any significant effect on control $I_{C a}$ with all of them peaking between -3.78 and -4.07 $\mathrm{pA} / \mathrm{pF}$ (Figure 5B, E, H, and K). However, nocodazole blunted the $I_{\mathrm{Ca}}$ response to ISO by $\sim 72 \%$ (1.18 \pm 0.08 -fold increase versus 1.65-fold change in untreated cells), halved the magnitude of leftward-shift of the voltage dependence of conductance $(6.73 \pm 1.38 \mathrm{mV}$ 
versus the $13.33 \mathrm{mV}$ shift in untreated cells), and eliminated the tendency toward cooperativity indicated by the slope of the Boltzmann function used to fit the $G / G_{\max }$ (Figure 5D-F; Table 1).

We used the same experimental paradigm to test whether the actin cytoskeleton plays any role in the functional regulation of $\mathrm{Cav} 1.2$ by $\beta A R \mathrm{~s}$. Cells treated with lat-A exhibited a $40 \%$ reduction in the $\mathrm{I}_{\mathrm{Ca}}$ augmentation response to ISO compared to controls (Figure 4G and $\mathrm{H}$, Table 1), and a similar halving of the leftward-shift in the voltage dependence of conductance as in nocodazole-treated cells (5.92 \pm 1.63 shift; Figure 5 I and Table 1). ISO stimulation produced $\mathrm{G} / \mathrm{G}_{\max }$ data that was well-fit with a Boltzmann function that was less steep than in ISO-stimulated untreated cells but still indicated enhanced cooperativity (Figure $5 \mathrm{I}$ and Table 1).

Finally, we examined the effect of ISO on $I_{\text {Ca }}$ when both MTs and actin filaments were disrupted with a combined nocodazole and lat-A treatment. Under these conditions, $\beta A R$ mediated regulation of Cav1.2 was essentially abolished (Figure 5J-K), with ISO, generating only a $1.01 \pm 0.11$-fold increase in $l_{\mathrm{Ca}}$, representing a $98 \%$ reduction in the response compared to untreated cells. The leftward-shift in the voltage dependence of conductance was reduced to $2.54 \pm 1.49$, equivalent to only $20 \%$ of the response seen in untreated cells. The slope factor of the Boltzmann-function used to fit the data was less steep than untreated cells in both control and ISO-stimulated conditions, indicating reduced cooperativity. These functional data collectively indicate that an intact cytoskeleton is an essential requirement for $\beta A R$-mediated regulation of Cav1.2.

\section{Clustering of Cav1.2 channels is supported by the cytoskeleton}

The observation that channel cooperativity was altered in AMVMs with disrupted cytoskeletal elements implies that the cytoskeleton might be important not only for $\beta A R$ mediated regulation of Cav1.2 but also for the stabilization and support of channel clusters. We tested this idea by examining Cav1.2 channel distribution with Ground $\underline{\text { State Depletion }}$ (GSD) super-resolution nanoscopy. ISO-stimulation resulted in the formation of Cav1.2 channel super-clusters in AMVMs which were, on average, 22.6\% larger than the clusters in control AMVMs (Figure 6A and E). Super-clustering could occur due to small clusters 
fusing together to form larger ones, or alternatively, could reflect enhanced sarcolemmal expression of $\mathrm{Cav} 1.2$. If the superclusters form because of enhanced insertion/exocytosis of Cav1.2 into the sarcolemma, then a testable prediction is that the intensity of the fluorescence emission (normalized to the cell area) should be increased. In contrast, if the super-clusters simply reflect fusion of existing sarcolemmal Cav1.2 clusters then the total fluorescence intensity should be similar in control and ISO-treated cells. Accordingly, the normalized total integrated density was significantly larger in ISO-treated cells than controls (Figure 6F; $P=0.00002$ ), in agreement with the idea that $\beta A R$-activation stimulates enhanced sarcolemmal insertion and resultant super-clustering of Cav1.2 channels. Cytoskeletal disruption with nocodazole (Figure 6B), lat-A (Figure 6C), or a combination of the two (Figure 6D), did not affect basal channel expression in the sarcolemma as indicated by similar cluster areas and total integrated density values (Figure 6E-F). These results validate the unaltered $I_{C a}$ observed in unstimulated cells from each of our four experimental groups (Figure 5) and supports the postulate that the lifetime of these channels in the membrane is longer than the $2 \mathrm{~h}$ cytoskeletal disruption period. In addition, ISO-stimulation failed to induce super-clustering or enhanced sarcolemmal expression of Cav1.2 in AMVMs (Figure 6E-F). Altogether, these data suggest that both intact MTs and actin are necessary for the formation of Cav1.2 super clusters in response to ISO stimulation. 


\section{Discussion}

The data presented in the present study provide the first report of an endosomal pool of Cav1.2 channels in cardiomyocytes that undergoes rapid, targeted mobilization to the sarcolemma in response to $\beta A R$-activation, effectively creating an 'on-demand' trafficking pathway to facilitate a positive inotropic response during fight-or-flight. We present six major new findings: 1) intracellular pools of Cav1.2 channels are present on EEs, REs, and in LEs and lysosomes in AMVMs; 2) $\beta A R$-activation triggers Cav1.2 mobilization from EEs and REs to the sarcolemma via Rab4a-dependent fast, and Rab11a-dependent slow recycling pathways; 3) Cav1.2 are often inserted or removed from the sarcolemma as large multi-channel clusters, rather than individual channels; 4) stimulated insertion of Cav1.2 channels occurs along MTs; 5) the endosomal pool of Cav1.2 is fueled by actindependent endocytosis; and finally, 6) adrenergic regulation of Cav1.2 is abrogated by cytoskeletal disruption and loss of this dynamic recycling response. On the basis of these data, we present a new working model (Figure 7) for $\beta A R$-regulation of cardiac Cav1.2 channels in which stimulated recycling of Cav1.2, from sub-sarcolemmal pools of Rab4a and Rab11a-positive endosomes, results in enhanced expression of Cav1.2 at the membrane of AMVMs. Resultant super-clustering and cooperative gating of Cav1.2 channels contributes to the enhanced $I_{\mathrm{Ca}}$ and inotropic response.

Our data revealed pools of intracellular Cav1.2 channels on EEA1 and Rab4-positive EEs, on Rab11 positive REs, and in Rab7 positive LEs and lysosomes. In response to ISO, EE and RE-localized channels underwent rapid recycling into the sarcolemma via the Rab4adependent fast recycling pathway and the slower, Rab11a recycling pathway. While this is the first report of stimulated recycling of a recruitable intracellular reservoir of Cav1.2 in cardiomyocytes, small GTPase choreographed-recycling of endosome-localized ion channel pools are well-known to play a role in fine-tuning cellular responses to various stimuli including $\beta A R$ stimulation. For example, in neurons, intracellular AMPA receptors (AMPAR) located on REs undergo Rab11-dependent recycling to the PM of dendritic spines in response to PKA-mediated phosphorylation of their GluA1 subunit at S845 downstream of $\beta_{2} A R$ stimulation (reviewed in $(37,38)$ ). In the collecting ducts of the kidney, vasopressin release initiates a $\mathrm{G}_{\mathrm{s}}$-coupled signaling cascade that triggers PKAmediated phosphorylation of aquaporin-2 (AQP2) at S256 and consequent recycling of 
AQP2 from Rab11-positive REs to the apical PM $(39,40)$. In the heart, acute stress initiates Rab11-dependent mobilization of endosomal reservoirs of SUR2-containing $\mathrm{K}_{\mathrm{ATP}}$ channels and of KCNQ1-containing REs to the sarcolemma (41-44). Similarly, here we report an endosomal pool of Cav1.2 channels that undergoes 'on-demand', stimulated recycling upon activation of $\beta A R \mathrm{~s}$, providing a functional reserve that drives ventricular inotropy during sympathetic stimulation.

Despite their fundamental importance in cardiac EC-coupling, the difficulty in visualizing and measuring Cav1.2 channel dynamics means there is a remarkable lack of information about Cav1.2 channel trafficking, endocytosis, and recycling in cardiomyocytes, with much of the published data focusing instead on data collected from heterologous expression systems. A previous study found that shRNA knockdown of Rab11a did not affect $\mathrm{Ba}^{2+}$ currents through Cav1.2 in HEK293 cells or neonatal mouse ventricular myocytes while Rab11b knockdown significantly increased $I_{B a}(21)$, suggesting that Rab11b plays a contrary role to its close family member Rab11a, and actually regulates constitutive Cav1.2 channel degradation. While we did not measure currents from tsA201 cells, it is reasonable to expect that currents should scale proportionally with the number of Cav1.2 channels in the membrane, since the whole cell current $\left(I_{C a}\right)$ is the product of the number of channels $(N)$, open probability $\left(P_{o}\right)$, and single-channel current $\left(i_{C a}\right)$, i.e., $I_{C a}=N \cdot i_{C a} \cdot P_{o}$. We found no evidence that knockdown of Rab11a function affected basal channel cluster density $(P=0.12)$ or number of channels in the membrane (measured with normalized mean grey values; $P=0.16$ ) (compared to same-day controls with no Rab overexpression), in agreement with the findings of Kamp et al. However, knockdown of Rab11a function reduced the degree of $\beta A R$-stimulated Cav1.2 insertion by $67 \%$. This confirms the role of Rab11a in $\beta A R$-stimulated channel insertion rather than degradation.

Our work on live, AAV9-Cav $\beta_{2 a}$-transduced AMVMs provides intriguing new insights into Cav1.2 channel trafficking, and captures the complex dynamics of these channels. We find that these channels are often inserted into the sarcolemma as entire pre-formed clusters at nucleation sites. Sometimes, repetitive insertions were seen to occur at an individual site, conjuring an image of Cav1.2-carrying endosomes queued up along MTs, anchored at a sarcolemmal delivery hub. Furthermore, channel endocytosis often appeared to occur via removal of entire clusters, while in other cases, a slower, gradual 
removal of channels suggested ongoing removal of individual channels. Our results at physiological temperature indicate that these insertion and removal events occur extremely rapidly and can be stimulated by activation of GPCR-signaling pathways. Activation of $\beta A R$ s was observed to increase the probability of channel insertion while activation of Angll receptors increased the probability of channel removal. These very scenarios were predicted in a recently published computer model designed to test the hypothesis that ion channel clustering occurs via a stochastic self-assembly process (45). Our data provides answers to the hypotheticals raised by that model. Informing that models parameters with the experimental data acquired in this study would be an interesting sequel.

One well-characterized facet of cardiac Cav1.2 channel trafficking is their targeted anterograde-delivery to the t-tubule membrane along BIN1-anchored MTs via the biosynthetic delivery pathway (34). Reduced levels of BIN1 in cardiomyocytes isolated from failing human hearts are associated with impaired Cav1.2 channel delivery and slower onset calcium transients (10). Here, we find that Cav1.2 channel recycling also occurs along MTs. Three independent lines of evidence support this conclusion. Firstly, MT disruption prevented ISO-stimulated mobilization of Cav1.2 from EE pools (Figure 3D). Secondly, MT disruption significantly reduced stimulated channel insertions in AAV9Cav $\beta_{2 a}$-paGFP transduced AMVMs (Figure 4F and Movie 2). Thirdly, ISO-stimulated Cav1.2 super-clustering was absent in nocodazole-treated AMVMs (Figure 6B and E). Our findings that basal Cav1.2 channel distribution and $I_{C a}$ amplitude were unaffected by $2 \mathrm{hr}$ nocodazole treatment suggests that channel lifetime in the membrane is longer than $2 \mathrm{hrs}$, so that channels delivered along intact MTs prior to depolymerization by the drug, still largely remained there (Figure 6B). This agrees with previous measurements of PM Cav1.2 channel half-times of $\sim 3 \mathrm{hrs}$, although this has never been directly measured in cardiomyocytes (12). However, despite negligible effects on basal channel expression and function, inhibition of MT polymerization significantly reduced ISO-stimulated responses, blunting $I_{C a}$ augmentation, and eliminating enhanced channel recycling and resultant super-clustering. Reduced MT polymerization occurs in human heart failure where stabilized MTs form a dense network in cardiomyocytes $(46,47)$. The lack of polymerization and potentially enhanced MT catastrophe rates, can result in traffic jams along MTs, leading to defective cargo delivery (46). Indeed, a previous study on live 
ventricular myocytes reported reduced delivery of $\mathrm{K}_{\mathrm{v}} 4.2$ and $\mathrm{K}_{\mathrm{v}} 4.3$ channels to the sarcolemma due to increased MT catastrophe rates upon addition of hydrogen peroxide or in the ROS-rich post-myocardial infarction environment (48). Failing and aging myocytes display reduced Cav1.2 responsivity to ISO $(49,50)$, thus future studies should examine whether loss of adrenergic responsivity of Cav1.2 in HF occurs due to impaired channel trafficking and recycling along MTs.

Actin polymerization has also been reported to be an important determinant of cardiac ion channel trafficking, notably of Cx43 (51). Although we failed to visualize cortical F-actin because of the vast amount of sarcomeric actin in AMVMs, we found that actin disruption with lat-A led to reduced: i) ISO-stimulated mobilization of Cav1.2 from EEs (Figure $3 \mathrm{H}$ ), and ii) augmentation of Cav1.2 channel expression in the PM, as indicated by superresolution GSD imaging experiments (Figure 6C) and live-cell TIRF experiments on AAV9$C a_{v} \beta_{2 a}-p a G F P$ transduced AMVMs (Figure 4E). Furthermore, lat-A significantly blunted adrenergic regulation of the channels assessed with whole cell patch clamp (Figure 5G-I and Table1). The degree of apparent channel insertions in response to ISO remained at a similar level to untreated cells suggesting that MTs, not actin, play the dominant role in channel delivery to the sarcolemma (Figure 4F). However, the pool of channels that was mobilized to the membrane in the presence of lat-A did not appear to belong to the EE pool, since colocalization between EEA1 and Cav1.2 channels was unchanged by ISO. In the Cx43 literature, it has been proposed that Cx43 cargo on its way to the sarcolemma from the golgi along MTs, pauses at actin 'rest-stops' before being handed off to additional MTs to complete its journey to the membrane $(51,52)$. It is possible that a similar 'rest stop' system exists for Cav1.2 channel delivery in cardiomyocytes and that lat-A mediated actin disruption and ISO-stimulation, releases this pool, allowing them to traffic to the sarcolemma along MTs. This intriguing hypothesis remains to be proven.

ISO-stimulation of AMVMs with a combined treatment with nocodazole and lat-A increased channel endocytosis as indicated by increased colocalization between EEs and Cav1.2 in Airyscan images (Figure 3J), and actually led to reduced channel expression in the sarcolemma in response to ISO (Figure 4E), while adrenergic regulation of the channel was completely eliminated (Figure $5 \mathrm{~J}-\mathrm{L}$ ). On the basis of our functional patch clamp data, it is tempting to speculate that $\beta A R$-mediated regulation of Cav1.2 is heavily dependent 
on this stimulated channel recycling pathway, however, ISO-stimulation is also known to induce endocytosis and subsequent fast, actin-dependent-recycling and resensitization of the receptors themselves (53-55). It is therefore possible that the lack of functional response is simply because of a lack of sarcolemmal $\beta A R$ expression, as internalized receptors cannot recycle back to the membrane (56). To investigate this possibility, we bypassed the receptors and stimulated adenylyl cyclase directly with forskolin and studied the effect on channel dynamics in AAV9-Cav $\beta_{2 a}$-paGFP transduced AMVMs using TIRF imaging (Supplemental Figure 4). Forskolin $(1 \mu \mathrm{M})$ produced a similar increase in sarcolemmal Cav $\beta_{2 a}$-paGFP expression (21.60 $\pm 3.72 \%$ ) as that observed with $100 \mathrm{nM}$ ISO $(27.95 \pm 4.31 \%)$. Treatment of AMVMs with cytoskeletal disruptors reduced the response (Figure S4B-E). These experiments do not totally rule out a direct $\beta A R$ effect however, as although it is GRK-mediated phosphorylation of $\beta A R s$ that increases their affinity for $\beta$-arrestin, subsequent movement into clathrin-coated pits and endocytosis; PKA-mediated desensitization of $\beta A R s$ can occur with forskolin (57). However, in a 2-3 minute exposure to forskolin or ISO, previous studies have revealed that only $20-30 \%$ of the receptors would be rendered desensitized (58), and this cannot explain our observed abolition of $\beta A R$-regulation of $I_{\mathrm{Ca}}$ (Figure 4), suggesting that agonist-stimulated recycling of Cav1.2 is in fact a critical component of $\beta A R$-regulation of these channels.

After several decades of elusivity, the critical PKA phosphorylation site on the cardiac Cav1.2 channel complex was recently reported to be located on Rad, a member of the $\mathrm{Rad} / \mathrm{Rem} / \mathrm{Rem} 2 / \mathrm{Gem} / \mathrm{Kir}$ (RGK) family of monomeric GTP-binding proteins that interacts with the channel via the $\beta$-subunit (5). In a disinhibition process, Rad phosphorylation is said to cause dissociation from the channel complex releasing its inhibitory hold on the channel, and unveiling the larger $I_{\mathrm{Ca}}$ we recognize as $\beta A R$-regulation. A Rad-mediated Cav1.2 disinhibition hypothesis was also proposed several years earlier by Jonathan Satin's group when they reported that Rad knockout mice lost adrenergic regulation (6). So how does our $\beta A R$-stimulated recycling of Cav1.2 fit into this appealing model? We do not believe the two models are mutually exclusive but instead hypothesize that Rad inhibits Cav1.2 channel function by limiting its expression at the sarcolemma, an effect that is relieved when Rad is phosphorylated by PKA. Indeed, in addition to the ability of Rad to suppress channel activity by interfering with channel $P_{\mathrm{o}}$, it has long been reported 
that RGK-proteins, including Rad, also reduce $I_{\mathrm{Ca}}$ by limiting Cav1.2 expression at the sarcolemma (59-61). A previous study in PC12 cells reported that this effect of Rad on PM Cav1.2 channel expression relied on CaM-binding to Rad, such that mutation of the CaM binding site at L281G abrogated the inhibitory effect of Rad on $I_{C a}$ and increased detection of a HA-tagged Cav1.2 channel at the cell surface (59). In cells transfected with WT Rad, the cell surface transport of Cav1.2 was blocked. Our results in transduced AMVMs, and in tsA cells, illustrate that ISO-stimulates enhanced transport of channels to the cell surface. We speculate that this transport occurs when Rad is phosphorylated and dislodges from $\operatorname{Cav} \beta$, releasing the channel complex and allowing more of it to traffic to the surface in a Rab4a and Rab11a dependent process. Indeed, that phosphorylation of Rad may be the 'upstream step' that has to occur before this recycling process is initiated, explaining why overexpression of CA-Rab4a does not raise the initial expression of Cav1.2 in the membrane in and of itself, but rather Rad phosphorylation must occur first. How PKA is anchored next to Rad on endosomes is another matter but may depend on the Akinase anchoring protein D-AKAP2, which has been reported to regulate recycling of transferrin receptors via interactions with Rab4 and Rab11 (62). In line with that prediction, a human functional polymorphism in D-AKAP2 (I646V) is associated with reduced heart rate variability, indicative of a heart that cannot respond well to stressors (63). D-AKAP2 itself can be phosphorylated by PKA at residue 554 (62) and this may influence its localization. We briefly tested the hypothesis that ISO-stimulation of $\beta A R s$ would promote enhanced colocalization between D-AKAP2 and Rab11-positive endosomes finding an enhanced association (Figure S5). While this is admittedly, a correlative result, resolving these mechanistic details will make for an interesting future project.

Overall, our data indicate that there is an endosomal pool of intracellular Cav1.2 channels that is stimulated to recycle to the sarcolemma upon $\beta A R$-activation. Both Rab4amediated fast recycling pathways, and Rab11a-slow recycling pathways contribute to the response. Recycling channels insert into the sarcolemma by traveling along MTs likely anchored at the PM by BIN1. Cytoskeletal disruption creates traffic jams in the endosomal pathway and impedes the recycling response. Finally, electrophysiology data indicates that $\beta A R$-regulation of cardiac Cav1.2 channel function is abolished by treatments that interfere with this stimulated recycling pathway. Collectively, these results suggest that cardiomyocytes have an endosomal reservoir of Cav1.2 channels that is rapidly mobilized 
bioRxiv preprint doi: https://doi.org/10.1101/2020.09.28.317362; this version posted September 28, 2020. The copyright holder for this preprint (which was not certified by peer review) is the author/funder, who has granted bioRxiv a license to display the preprint in perpetuity. It is made available under aCC-BY-NC-ND 4.0 International license.

to the sarcolemma in response to $\beta A R$-stimulation, and that this stimulated insertion is fundamentally required for $\beta A R$-regulation of these channels. 


\section{Materials and Methods}

Detailed methods can be found in the SI Appendix. Briefly, AMVMs were enzymatically isolated using standard Langendorff technique as described previously $(23,64,65)$. Fixed, immunostained AMVMs were imaged on a Zeiss Airyscan confocal microscope (as described in $(66,67)$ ) or a Leica 3D-GSD-SR microscope to assess the distribution of Cav1.2 channels and various endosome populations (Airyscan only for the latter). Live cell TIRF imaging experiments of AAV9-Cav $\beta_{2 a}$-paGFP transduced AMVMs or transiently transfected tsA-201 cells were performed on an Olympus IX-83 inverted microscope with a Cell-TIRF MITICO module. Live cell 4D-imaging of transduced AMVMs was performed at $37^{\circ} \mathrm{C}$ on an Andor W-1 Spinning Disk confocal microscope. Rab mutant plasmids used for transient transfection of tsA-201 cells were gifts from Dr. Nipavan Chiamvimonvat (UC Davis, Davis, CA, USA) and Dr. Jose A. Esteban (Centro de Biología Molecular 'Severo Ochoa' (CSIC-UAM), Madrid, Spain).

\section{Acknowledgments}

We wish to thank Dr. Luis Fernando Santana for the use of his GSD microscope, and for reading and commenting on this manuscript along with Dr. Manuel F. Navedo, and Dr. Maartje Westhoff.

\section{Funding}

This work was supported by NIH NIA grant R01AG063796 and AHA grant 15SDG25560035 to RED and an NIGMS grant R01GM127513 to EJD. Taylor Voelker and Heather Spooner were supported by a NIGMS-funded Pharmacology Training Program (T32GM099608). 
Figures and Tables

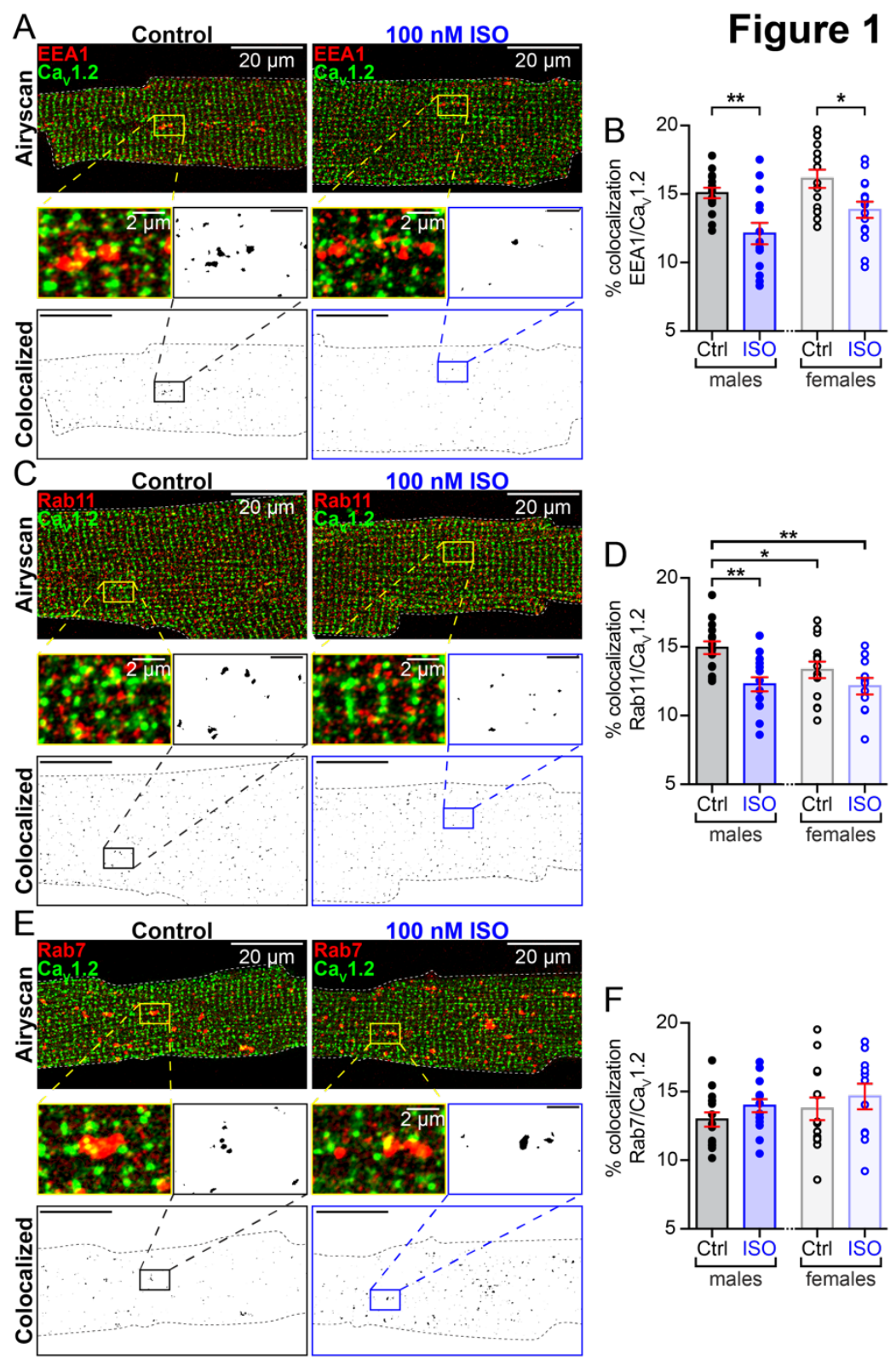

Figure 1. Internal pools of presynthesized Cav1.2 channels reside on endosomes.

(A) Two-color Airyscan super-resolution images of control and $100 \mathrm{nM}$ ISO-stimulated, AMVMs immunostained to examine distributions of Cav1.2 and EEA1 positive early endosomes. Binary colocalization maps (bottom) display pixels in which Cav1.2 and endosomal expression precisely overlapped. $(B)$ Histograms showing \% colocalization between EEA1 and Cav1.2 in male and female AMVMs, in control (male: $N=3, n=15$; female $N=3, n=13$ ) and ISO-stimulated conditions (male: $N=3, n=14$; female $N=3, n$ = 15). (C) Immunostaining of Cav1.2 and Rab11 positive recycling endosomes and $(D)$ 
accompanying histogram summarizing results from control (male: $N=4, n=15$; female $N$ $=3, n=14$ ) and ISO conditions (male: $N=3, n=15$; female $N=3, n=11)$. $(E)$ Immunostaining of Cav1.2 and Rab7 positive LEs and lysosomes. (F) Histogram summarizing results from control (males: $N=3, n=15$, females: $N=3, n=14$ ) and ISOstimulated AMVMs (males: $N=3, n=15$, females: $\mathrm{N}=3, \mathrm{n}=11$ ). Error bars indicate SEM. Two-way ANOVA ${ }^{* *} \mathrm{P}<0.01 ;{ }^{*} \mathrm{P}<0.05$. Pictured are male AMVMs, for females see Figure S1. 


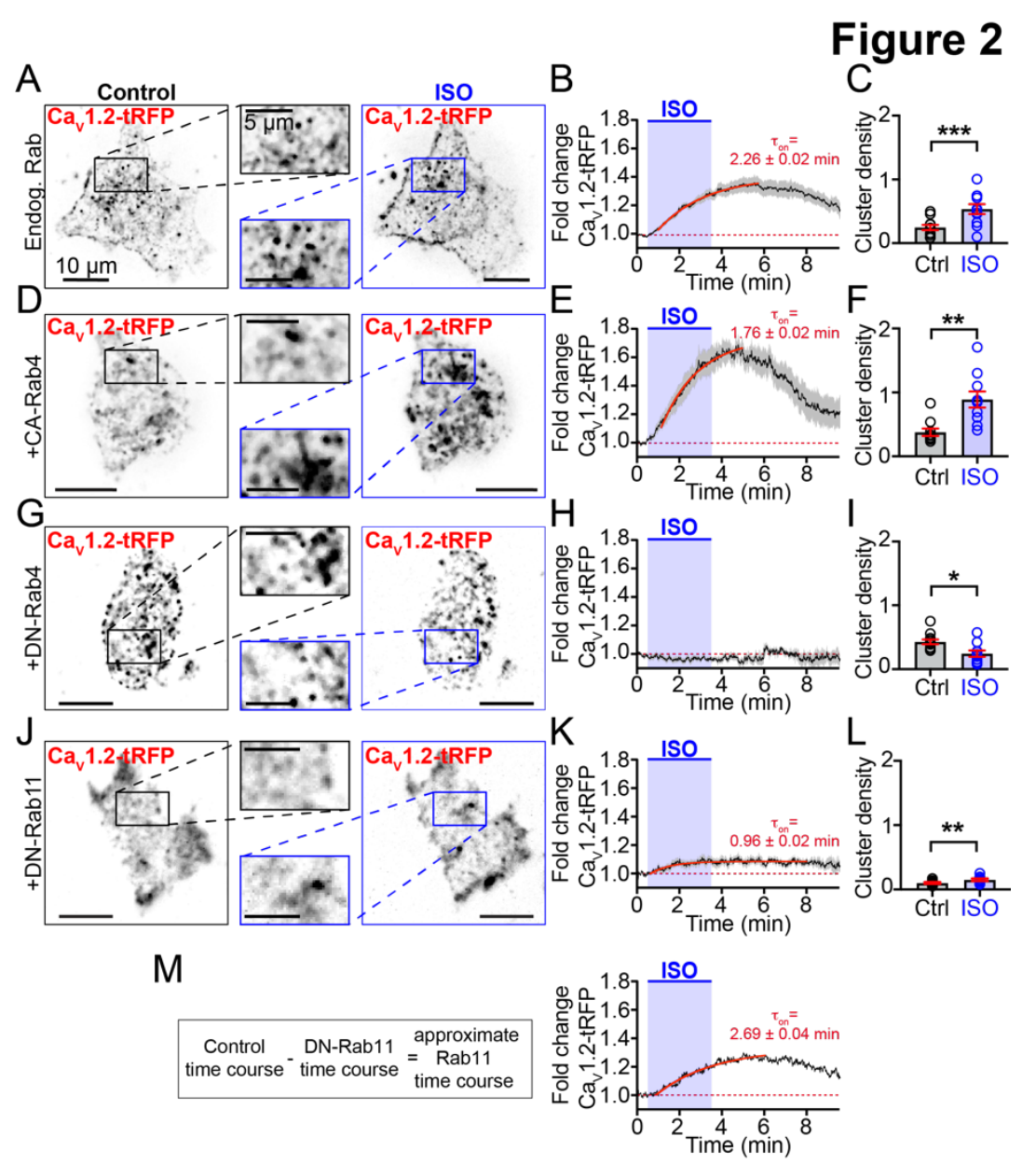

Figure 2. Rab4a and Rab11a regulate an ISO-stimulated boost in Cav1.2 recycling.

(A) TIRF images of Cav1.2-tRFP distribution in the PM of tsA-201 cells before (left) and after $100 \mathrm{nM}$ ISO (right; $n=18$ ). (B) Time course and kinetics (solid red line) of the fold change in Cav1.2-tRFP intensity in the TIRF footprint before, during and after ISO stimulation. (C) Histogram summarizing Cav1.2 channel cluster density (number/ $\mu m^{2}$ ) in the TIRF footprint in control and ISO stimulated conditions. (D- $F$ ) Same layout format in tsA-201 cells co-expressing constitutively active (GTP-locked) Rab4a ( $n=10)$. (G- $l$ ) Same layout format in tsA-201 cells co-expressing dominant negative (GDP-locked) Rab4a ( $n=$ 12). (J-L) Same layout format in tsA-201 cells co-expressing dominant negative (GDPlocked) Rab11a $(n=8)$. (M) Calculation and resultant theoretical time-course and kinetics of Rab11a-dependent ISO-stimulated recycling. 


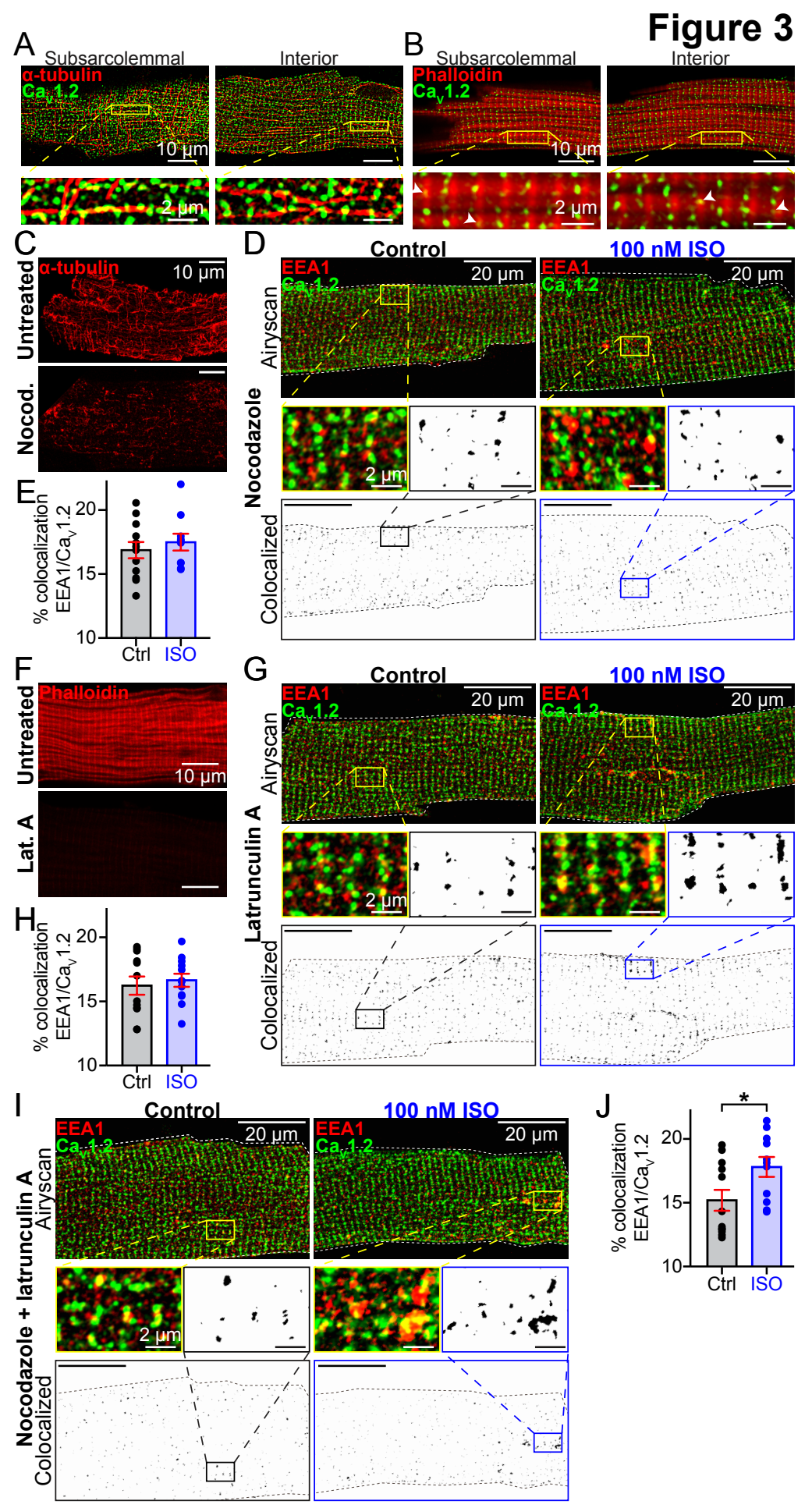

Figure 3. Actin and MT polymerization are essential for ISO-stimulated Cav1.2 recycling. Two-color Airyscan images of fixed AMVMs immunostained to examine the relative localization of Cav1.2 and $(A)$ a-tubulin, or $(B)$ phalloidin-stained actin. $(C)$ shows the distribution of a-tubulin in untreated (top) and nocodazole-treated AMVMs (bottom). 
(D) Airyscan images of Cav1.2 and EEA1 distribution in nocodazole-treated control $(N=$ 3, $n=12$; left) and ISO-stimulated ( $N=3, n=10$; right) AMVMs. Bottom: binary colocalization maps display pixels in which Cav1.2 and EEA1 expression precisely overlapped. ( $E$ ) Histogram summarizing \% colocalization of EEA1 with Cav1.2 in nocodazole-treated cells. ( $F$ ) Actin distribution in untreated (top) and latrunculin-A treated cells (bottom). (G) Airyscan images and binary colocalization maps of Cav1.2 and EEA1 distribution in latrunculin-A-treated control $(N=3, n=12$; left $)$ and ISO-stimulated $(N=3$, $n=12$; right) AMVMs. $(H)$ Histogram showing \% colocalization of EEA1 with Cav1.2 in latrunculin-A-treated AMVMs. (I) Airyscan images and binary colocalization maps of AMVMs treated with both latrunculin-A and nocodazole under control $(N=3, n=12)$ and ISO stimulated conditions $(N=3, n=11)$, with accompanying summary histogram ( $)$. 
A
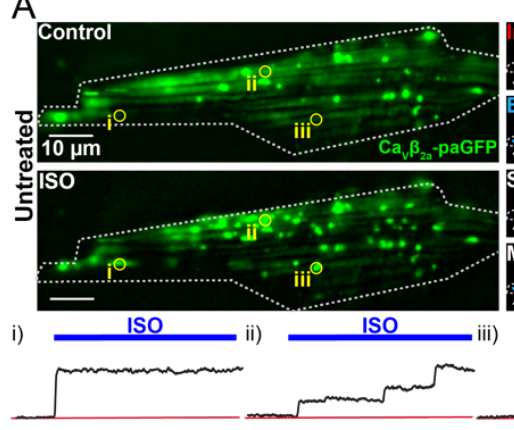

C
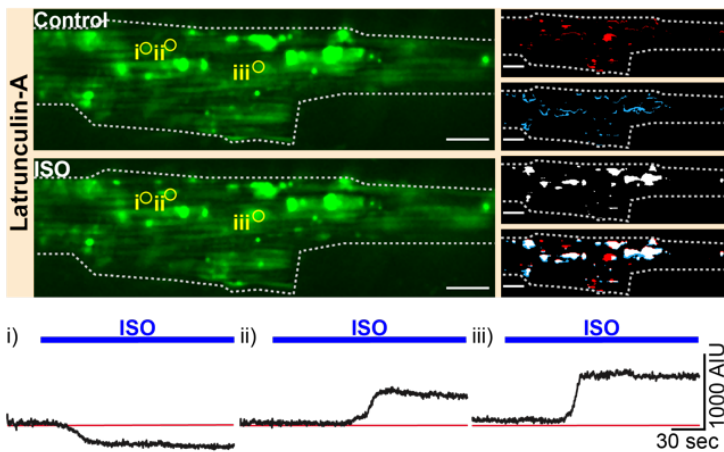

$\mathrm{E}$

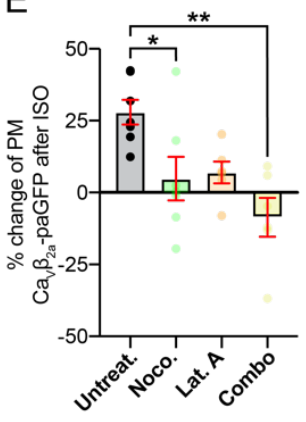

$\mathrm{F}$

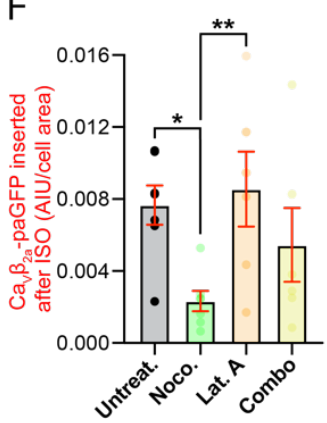

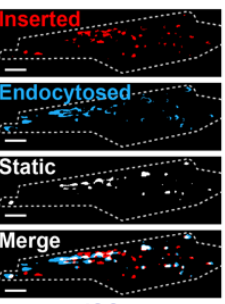

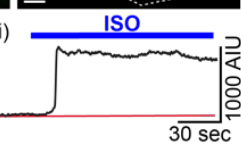

$\mathrm{B}$
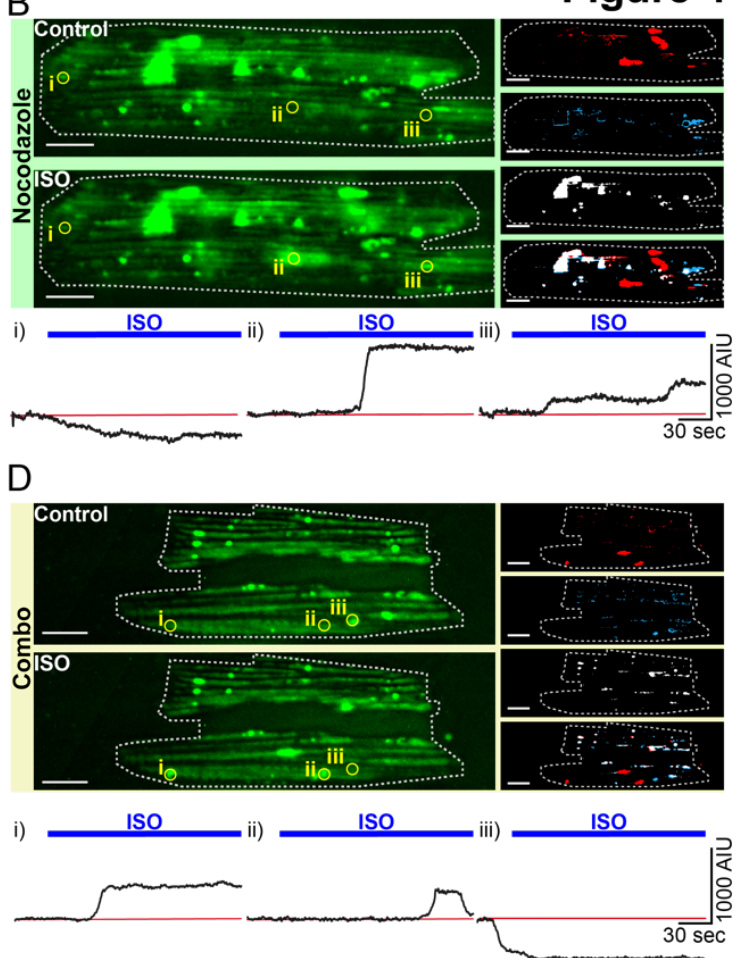

G

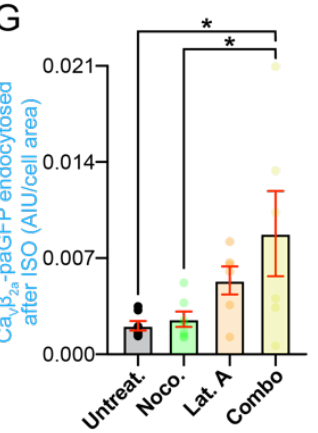

Figure 4
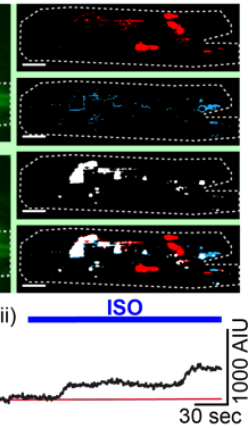

$\mathrm{H}$

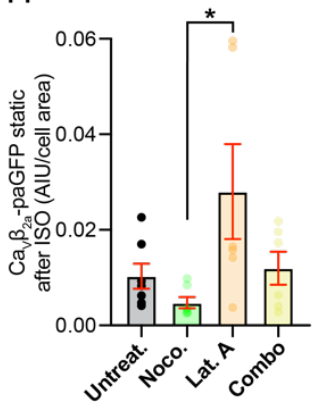

Figure 4. Dynamic imaging to unmask the mobile channel population. $(A)$ TIRF images of GFP fluorescence emission from $\mathrm{Ca}_{v} \beta_{2 a}$-paGFP transduced AMVMs before (top) and after $100 \mathrm{nM}$ ISO (bottom). Images illustrating inserted, endocytosed, static, and merged channel populations are shown to the right. Bottom: Time course of the changes in $\mathrm{Ca}_{v} \beta_{2 a}$-paGFP intensity in ROIs (i-iii) indicated by yellow circles on TIRF images. $(N=$ $5, n=7)$. Same format for cells pre-treated with $(B)$ nocodazole $(N=5, n=7),(C)$ latrunculin-A $(N=3, n=6)$, or $(D)$ both cytoskeleton disruptors $(N=3, n=6)$. $(E-H)$ Histograms summarizing statistics for these experiments. 


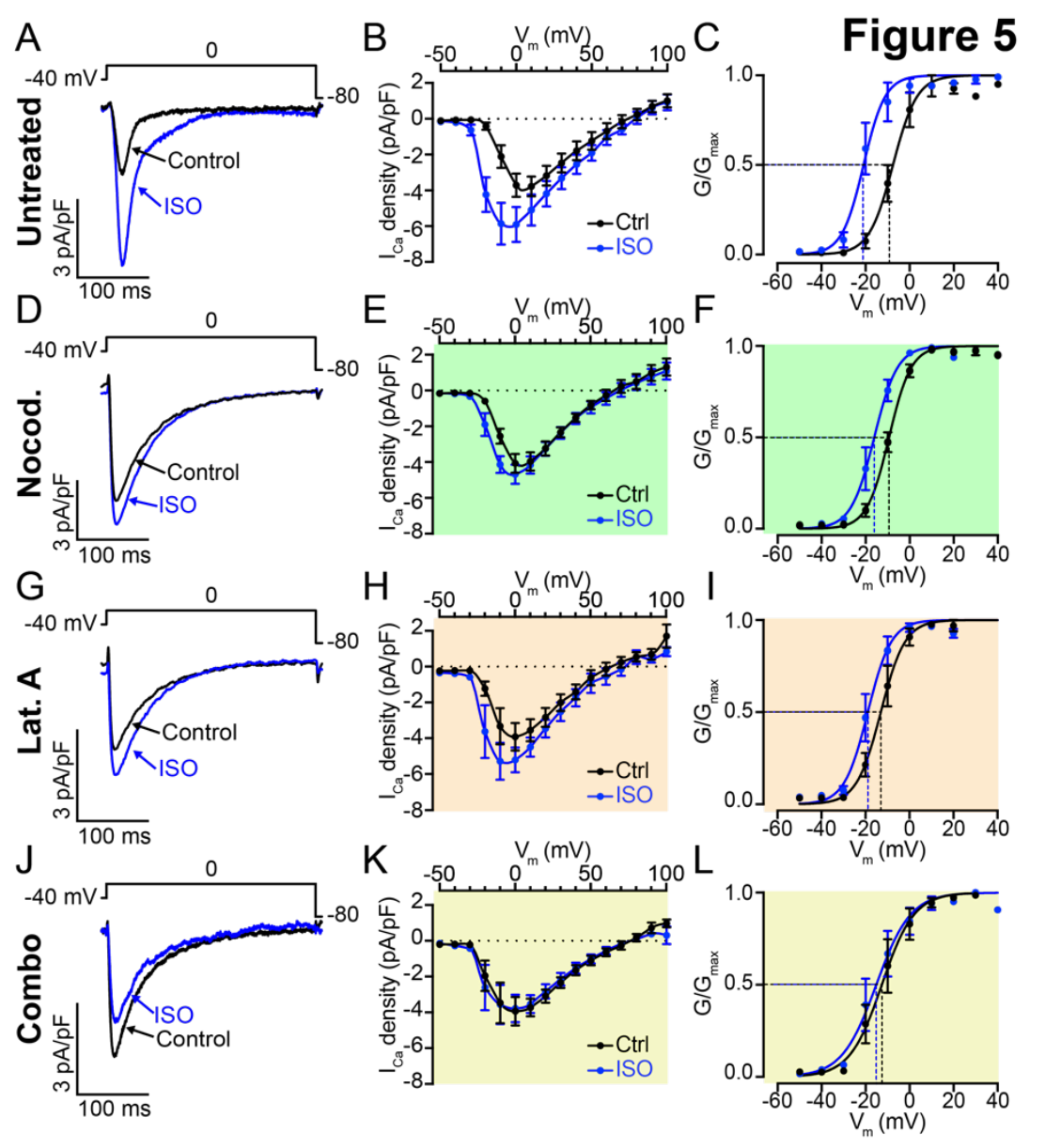

Figure 5. $\beta A R$ mediated $\mathrm{I}_{\mathrm{Ca}}$ enhancement is blunted by reducing dynamic channel insertion. $(A)$ Whole cell currents elicited from a representative AMVM during a $300 \mathrm{~ms}$ depolarization step from $-40 \mathrm{mV}$ to $0 \mathrm{mV}$ before (control: black) and during application of $100 \mathrm{nM}$ ISO (blue). (B) I-V plot summarizing the results from $n=7$ cells (from $N=7$ animals) subjected to test potentials ranging from $-50 \mathrm{mV}$ to $+100 \mathrm{mV}$. Currents were normalized to cell capacitance to generate current density. $(C)$ Voltage-dependence of the normalized conductance ( $\mathrm{G} / \mathrm{G}_{\max }$ ) before and during ISO application, fit with Boltzmann functions. ( $D-F)$ show whole cell currents $(D), \mathrm{I}-\mathrm{V}(E)$, and $\mathrm{G} / \mathrm{G}_{\max }$ plots $(F)$ from AMVMs pre-treated for 2 hrs with $10 \mu \mathrm{M}$ nocodazole $(N=4, n=7)$. ( $G-H)$ show the same for cells treated with $5 \mu \mathrm{M}$ lat-A $(N=5, n=5)$. $(J-L)$ show results from cells treated with a combination of both $(N=3, n=6)$. 
A

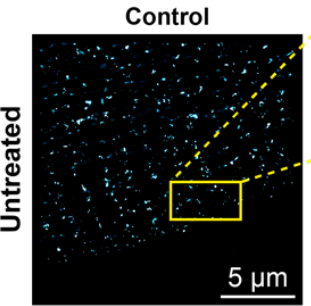

B

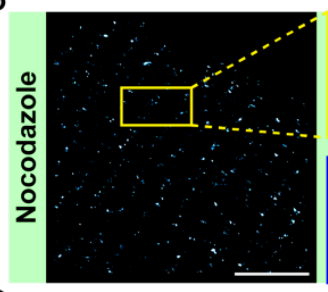

C

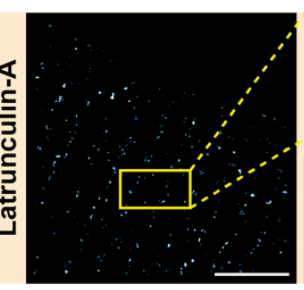

D
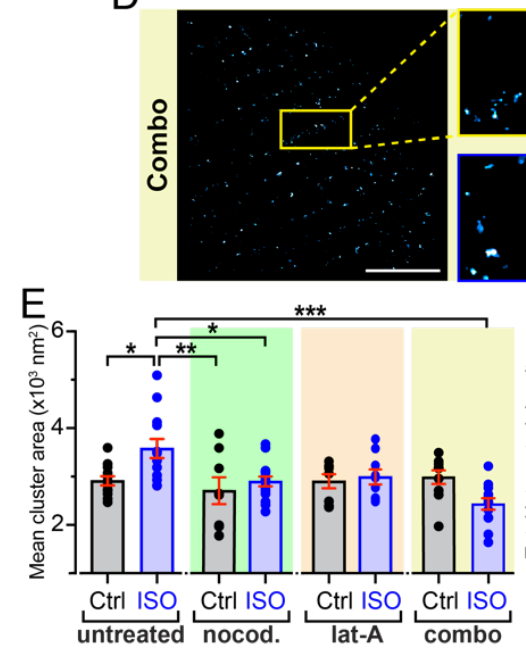

Figure 6
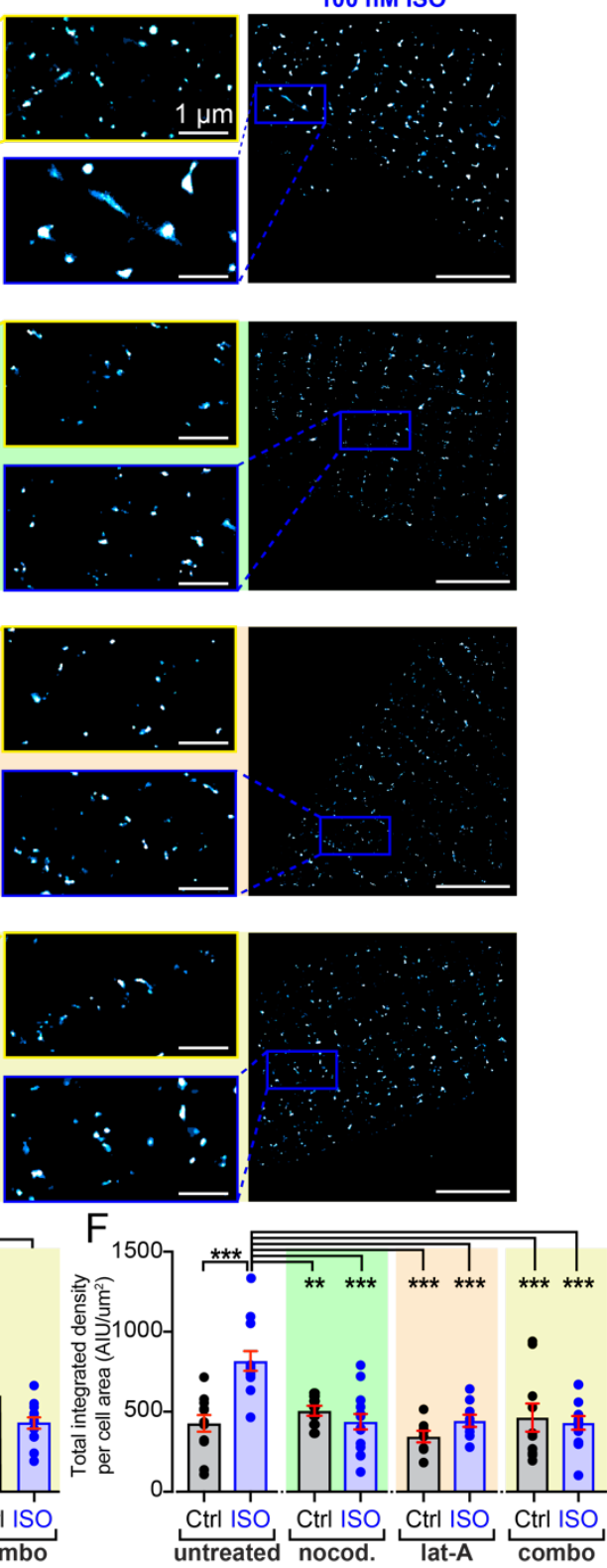

Figure 6. $\beta A R$-stimulated Cav1.2 super-clustering and enhanced sarcolemmal expression requires an intact MT cytoskeleton. Super-resolution GSD localization maps of control (left) and $100 \mathrm{~nm}$ ISO-stimulated (right), fixed, AMVMs immunostained to examine Cav1.2 channel distribution under $(A)$ untreated (control: $N=3, n=13$; ISO: $N=$ 3, $n=13)$, (B) nocodazole-treated (control: $N=3, n=8$; ISO: $(N=3, n=15)$, (C) lat-Atreated (control: $N=3, n=8$; ISO: $(N=3, n=9)$, and $(D)$ combo-treated (control: $N=2, n$ = 10; ISO: $(N=3, n=13)$ conditions. Maps were pseudocoloured 'cyan hot' and received a one-pixel median filter for display purposes. Yellow (control) and blue (ISO) boxes 
bioRxiv preprint doi: https://doi.org/10.1101/2020.09.28.317362; this version posted September 28, 2020. The copyright holder for this preprint (which was not certified by peer review) is the author/funder, who has granted bioRxiv a license to display the preprint in perpetuity. It is made available under aCC-BY-NC-ND 4.0 International license.

indicate the location of the zoomed-in regions displayed in the center. $(E)$ and $(F)$, aligned dot plots showing mean Cav1.2 channel cluster areas and normalized total integrated density in each condition. Two-way ANOVA ${ }^{* *} \mathrm{P}<0.001$, ${ }^{* *} \mathrm{P}<0.01,{ }^{*} \mathrm{P}<0.05$. 


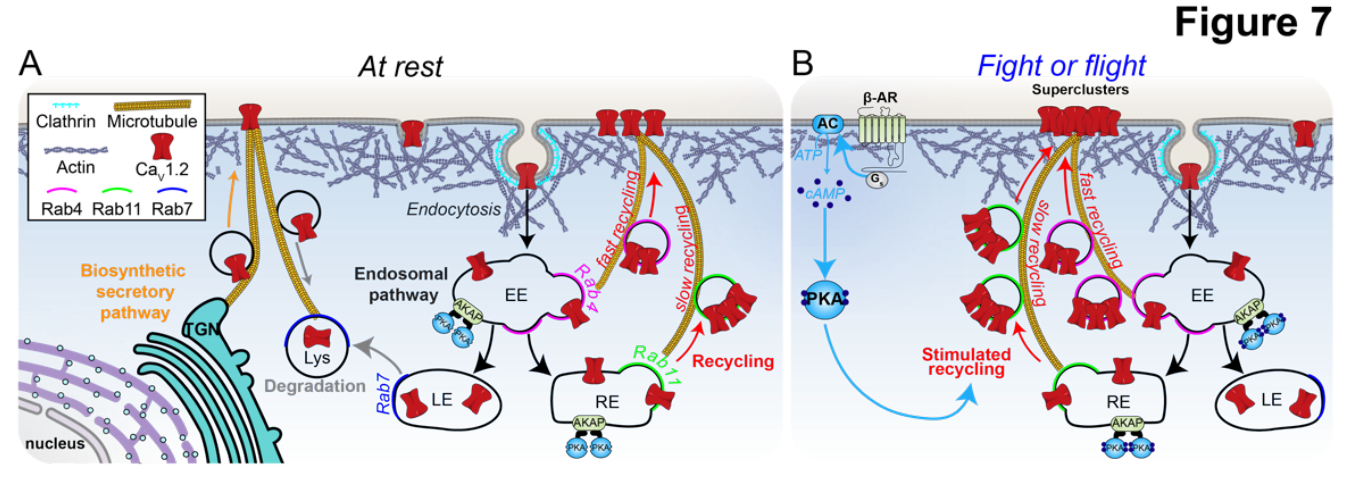

Figure 7. Our working model. A: Cav1.2 channels on the ventricular myocyte sarcolemma are subject to dynamic equilibrium under resting, unstimulated conditions. Channels synthesized in the Golgi are transferred to negative-end anchored MTs at the trans-Golgi network (TGN) and transported in motor-protein shuttled vesicles, to plus-end anchored BIN1 hubs at the membrane. Steady state channel expression is achieved as 'new' channel insertions are balanced by ongoing channel endocytosis and recycling through the endocytic pathway. $B$ : Activation of $\beta A R$ s by ISO or during fight or flight, leads to stimulated recycling of Cav1.2, from sub-sarcolemmal pools of Rab4a and Rab11apositive endosomes. D-AKAP2 anchors PKA on endosomal membranes, future studies should investigate its role in $\beta A R$-stimulated channel recycling. Resultant 'on-demand' insertion of the endosomal reservoir of Cav1.2, leads to augmented Cav1.2 expression and super-clustering at the sarolemma, facilitates channel cooperativity, amplifies $\mathrm{Ca}^{2+i n f l u x}$, and contributes to the enhanced $I_{\mathrm{Ca}}$ and inotropic response. 
bioRxiv preprint doi: https://doi.org/10.1101/2020.09.28.317362; this version posted September 28. 2020. The copvriaht holder for this preprint (which was not certified by peer review) is the author/funder, who has granted bioRxiv a license to display the preprint in perpetuity. It is made available under aCC-BY-NC-ND 4.0 International license.

\section{Table 1}

\begin{tabular}{|c|c|c|c|c|c|}
\hline & \multirow[b]{2}{*}{ Fold-change in peak $\mathrm{I}_{\mathrm{Ca}}$} & \multicolumn{2}{|c|}{$\mathrm{V}_{1 / 2}(\mathrm{mV})$} & \multicolumn{2}{|c|}{ Slope factor } \\
\hline & & Control & ISO & Control & ISO \\
\hline Untreated & $1.65 \pm 0.19^{*}$ & $-7.57 \pm 0.95$ & $-20.90 \pm 1.14^{\star \star \star \star}$ & $5.45 \pm 0.84$ & $4.90 \pm 1.06$ \\
\hline Nocodazole & $1.18 \pm 0.08$ & $-9.38 \pm 0.44$ & $-16.10 \pm 0.84^{\star \star \star}$ & $5.03 \pm 0.41$ & $5.27 \pm 0.72$ \\
\hline Latrunculin A & $1.39 \pm 0.13$ & $-13.03 \pm 0.87$ & $-18.95 \pm 0.97^{\star}$ & $5.51 \pm 0.76$ & $5.33 \pm 0.88$ \\
\hline Noco + Lat.A & $1.01 \pm 0.11$ & $-12.62 \pm 1.40$ & $-15.16 \pm 1.53$ & $7.32 \pm 1.24$ & $7.82 \pm 1.36$ \\
\hline
\end{tabular}




\section{References}

1. S. Ringer, A further Contribution regarding the influence of the different Constituents of the Blood on the Contraction of the Heart. J Physiol 4, 29-42 23 (1883).

2. H. Reuter, H. Scholz, The regulation of the calcium conductance of cardiac muscle by adrenaline. J Physiol 264, 49-62 (1977).

3. D. T. Yue, S. Herzig, E. Marban, $\beta$-adrenergic stimulation of calcium channels occurs by potentiation of high-activity gating modes. Proc Natl Acad Sci U S A 87, 753-757 (1990).

4. N. Sperelakis, J. A. Schneider, A metabolic control mechanism for calcium ion influx that may protect the ventricular myocardial cell. The American journal of cardiology 37, 1079-1085 (1976).

5. G. Liu et al., Mechanism of adrenergic Cav1.2 stimulation revealed by proximity proteomics. Nature 577, 695-700 (2020).

6. J. R. Manning et al., Rad GTPase deletion increases L-type calcium channel current leading to increased cardiac contraction. J Am Heart Assoc 2, e000459 (2013).

7. X. Chen et al., L-type $\mathrm{Ca}^{2+}$ channel density and regulation are altered in failing human ventricular myocytes and recover after support with mechanical assist devices. Circ Res 91, 517-524 (2002).

8. J. He et al., Reduction in density of transverse tubules and L-type $\mathrm{Ca}^{2+}$ channels in canine tachycardia-induced heart failure. Cardiovascular research 49, 298-307 (2001).

9. I. R. Josephson, A. Guia, M. D. Stern, E. G. Lakatta, Alterations in properties of L-type $\mathrm{Ca}^{2+}$ channels in aging rat heart. J Mol Cell Cardiol 34, 297-308 (2002).

10. T. T. Hong et al., BIN1 is reduced and Cav1.2 trafficking is impaired in human failing cardiomyocytes. Heart Rhythm 9, 812-820 (2012).

11. I. Pranke, A. Golec, A. Hinzpeter, A. Edelman, I. Sermet-Gaudelus, Emerging Therapeutic Approaches for Cystic Fibrosis. From Gene Editing to Personalized Medicine. Front Pharmacol 10, 121 (2019).

12. A. J. Chien et al., Roles of a membrane-localized beta subunit in the formation and targeting of functional L-type $\mathrm{Ca}^{2+}$ channels. J Biol Chem 270, 30036-30044 (1995).

13. R. Conrad et al., Rapid Turnover of the Cardiac L-Type Cav1.2 Channel by Endocytic Recycling Regulates Its Cell Surface Availability. iScience 7, 1-15 (2018).

14. W. C. Claycomb et al., HL-1 cells: a cardiac muscle cell line that contracts and retains phenotypic characteristics of the adult cardiomyocyte. Proc Natl Acad Sci U S A 95, 2979-2984 (1998).

15. A. Wandinger-Ness, M. Zerial, Rab proteins and the compartmentalization of the endosomal system. Cold Spring Harb Perspect Biol 6, a022616 (2014).

16. P. Buda et al., Eukaryotic translation initiation factor 3 subunit e controls intracellular calcium homeostasis by regulation of Cav1.2 surface expression. PLoS One 8, e64462 (2013).

17. H. Stenmark, Rab GTPases as coordinators of vesicle traffic. Nat Rev Mol Cell Biol 10, 513-525 (2009).

18. E. Mizuno-Yamasaki, F. Rivera-Molina, P. Novick, GTPase networks in membrane traffic. Annual review of biochemistry 81, 637-659 (2012).

19. F. R. Maxfield, T. E. McGraw, Endocytic recycling. Nat Rev Mol Cell Biol 5, 121-132 (2004).

20. E. M. Green, C. F. Barrett, G. Bultynck, S. M. Shamah, R. E. Dolmetsch, The tumor suppressor elF3e mediates calcium-dependent internalization of the L-type calcium channel Cav1.2. Neuron 55, 615-632 (2007). 
21. J. M. Best et al., Small GTPase Rab11b regulates degradation of surface membrane L-type Cav1.2 channels. Am J Physiol Cell Physiol 300, C1023-1033 (2011).

22. D. Ghosh et al., Dynamic L-type Cav1.2 channel trafficking facilitates Cav1.2 clustering and cooperative gating. Biochimica et biophysica acta 1865, 1341-1355 (2018).

23. D. W. Ito et al., $\beta$-adrenergic-mediated dynamic augmentation of sarcolemmal Cav1.2 clustering and co-operativity in ventricular myocytes. J Physio/597, 2139-2162 (2019).

24. B. K. Atwood, J. Lopez, J. Wager-Miller, K. Mackie, A. Straiker, Expression of G protein-coupled receptors and related proteins in HEK293, AtT20, BV2, and N18 cell lines as revealed by microarray analysis. BMC Genomics 12, 14 (2011).

25. M. Cormont et al., Potential role of Rab4 in the regulation of subcellular localization of Glut4 in adipocytes. Molecular and cellular biology 16, 6879-6886 (1996).

26. S. C. Calaghan, J. Y. Le Guennec, E. White, Cytoskeletal modulation of electrical and mechanical activity in cardiac myocytes. Prog Biophys Mol Biol 84, 29-59 (2004).

27. I. Fujiwara, M. E. Zweifel, N. Courtemanche, T. D. Pollard, Latrunculin A Accelerates Actin Filament Depolymerization in Addition to Sequestering Actin Monomers. Curr Biol 28, 3183-3192 e3182 (2018).

28. I. Spector, N. R. Shochet, Y. Kashman, A. Groweiss, Latrunculins: novel marine toxins that disrupt microfilament organization in cultured cells. Science 219, 493-495 (1983).

29. W. E. Louch, K. A. Sheehan, B. M. Wolska, Methods in cardiomyocyte isolation, culture, and gene transfer. J Mol Cell Cardiol 51, 288-298 (2011).

30. H. C. Hartzell, P. F. Mery, R. Fischmeister, G. Szabo, Sympathetic regulation of cardiac calcium current is due exclusively to cAMP-dependent phosphorylation. Nature 351, 573-576 (1991).

31. J. Leroy et al., Spatiotemporal dynamics of $\beta$-adrenergic cAMP signals and L-type $\mathrm{Ca}^{2+}$ channel regulation in adult rat ventricular myocytes: role of phosphodiesterases. Circ Res 102, 1091-1100 (2008).

32. T. Hermosilla et al., Prolonged AT1R activation induces Cav1.2 channel internalization in rat cardiomyocytes. Sci Rep 7, 10131 (2017).

33. B. D. Grant, J. G. Donaldson, Pathways and mechanisms of endocytic recycling. Nat Rev Mol Cell Biol 10, 597-608 (2009).

34. T. T. Hong et al., BIN1 localizes the L-type calcium channel to cardiac T-tubules. PLoS Biol 8, e1000312 (2010).

35. D. D. Hall et al., Competition between alpha-actinin and $\mathrm{Ca}^{2+-}$-calmodulin controls surface retention of the L-type $\mathrm{Ca}^{2+}$ channel Cav1.2. Neuron 78, 483-497 (2013).

36. P. Y. Tseng et al., alpha-Actinin Promotes Surface Localization and Current Density of the $\mathrm{Ca}^{2+}$ Channel Cav1.2 by Binding to the IQ Region of the $\mathrm{a}_{1}$ Subunit. Biochemistry 56, 3669-3681 (2017).

37. G. H. Diering, R. L. Huganir, The AMPA Receptor Code of Synaptic Plasticity. Neuron 100, 314-329 (2018).

38. A. M. Purkey, M. L. Dell'Acqua, Phosphorylation-Dependent Regulation of $\mathrm{Ca}^{2+}$ Permeable AMPA Receptors During Hippocampal Synaptic Plasticity. Front Synaptic Neurosci 12, 8 (2020).

39. P. I. Nedvetsky et al., A Role of myosin Vb and Rab11-FIP2 in the aquaporin-2 shuttle. Traffic 8, 110-123 (2007).

40. K. Fushimi, S. Sasaki, F. Marumo, Phosphorylation of serine 256 is required for cAMPdependent regulatory exocytosis of the aquaporin-2 water channel. J Biol Chem $\mathbf{2 7 2}$, 14800-14804 (1997). 
41. L. Bao, K. Hadjiolova, W. A. Coetzee, M. J. Rindler, Endosomal KATP channels as a reservoir after myocardial ischemia: a role for SUR2 subunits. Am J Physiol Heart Circ Physiol 300, H262-270 (2011).

42. M. Jiang, Y. Wang, G. N. Tseng, Adult Ventricular Myocytes Segregate KCNQ1 and KCNE1 to Keep the $\mathrm{I}_{\mathrm{Ks}}$ Amplitude in Check Until When Larger IKs Is Needed. Circ Arrhythm Electrophysiol 10 (2017).

43. Y. Wang et al., $\left[\mathrm{Ca}^{2+}\right]_{i}$ elevation and oxidative stress induce $\mathrm{KCNQ} 1$ protein translocation from the cytosol to the cell surface and increase slow delayed rectifier (IKs) in cardiac myocytes. J Biol Chem 288, 35358-35371 (2013).

44. G. Seebohm et al., Regulation of endocytic recycling of KCNQ1/KCNE1 potassium channels. Circ Res 100, 686-692 (2007).

45. D. Sato et al., A stochastic model of ion channel cluster formation in the plasma membrane. J Gen Physiol 10.1085/jgp.201912327 (2019).

46. M. A. Caporizzo, C. Y. Chen, B. L. Prosser, Cardiac microtubules in health and heart disease. Exp Biol Med (Maywood) 244, 1255-1272 (2019).

47. C. Y. Chen et al., Suppression of detyrosinated microtubules improves cardiomyocyte function in human heart failure. Nature medicine 24, 1225-1233 (2018).

48. B. M. Drum et al., Oxidative stress decreases microtubule growth and stability in ventricular myocytes. J Mol Cell Cardiol 93, 32-43 (2016).

49. H. Ouadid, B. Albat, J. Nargeot, Calcium currents in diseased human cardiac cells. Journal of cardiovascular pharmacology 25, 282-291 (1995).

50. J. B. Strait, E. G. Lakatta, Aging-Associated Cardiovascular Changes and Their Relationship to Heart Failure. Heart Failure Clinics 8, 143-+ (2012).

51. J. W. Smyth et al., Actin cytoskeleton rest stops regulate anterograde traffic of connexin 43 vesicles to the plasma membrane. Circ Res 110, 978-989 (2012).

52. S. S. Zhang, S. Hong, A. G. Kleber, L. P. Lee, R. M. Shaw, A micropatterning approach for imaging dynamic Cx43 trafficking to cell-cell borders. FEBS Lett 588, 1439-1445 (2014).

53. A. C. Hanyaloglu, M. von Zastrow, Regulation of GPCRs by endocytic membrane trafficking and its potential implications. Annual review of pharmacology and toxicology 48, 537-568 (2008).

54. R. Irannejad et al., Conformational biosensors reveal GPCR signalling from endosomes. Nature 495, 534-538 (2013).

55. G. A. Yudowski, M. A. Puthenveedu, A. G. Henry, M. von Zastrow, Cargo-mediated regulation of a rapid Rab4-dependent recycling pathway. Mol Biol Cell 20, 2774-2784 (2009).

56. E. E. Millman et al., Rapid recycling of beta-adrenergic receptors is dependent on the actin cytoskeleton and myosin Vb. Traffic 9, 1958-1971 (2008).

57. J. Pitcher, M. J. Lohse, J. Codina, M. G. Caron, R. J. Lefkowitz, Desensitization of the isolated $\beta_{2}$-adrenergic receptor by $\beta$-adrenergic receptor kinase, cAMP-dependent protein kinase, and protein kinase $\mathrm{C}$ occurs via distinct molecular mechanisms. Biochemistry 31, 3193-3197 (1992).

58. N. S. Roth, P. T. Campbell, M. G. Caron, R. J. Lefkowitz, M. J. Lohse, Comparative rates of desensitization of $\beta$-adrenergic receptors by the $\beta$-adrenergic receptor kinase and the cyclic AMP-dependent protein kinase. Proc Natl Acad Sci U S A 88, 62016204 (1991).

59. P. Beguin et al., Nuclear sequestration of beta-subunits by Rad and Rem is controlled by 14-3-3 and calmodulin and reveals a novel mechanism for $\mathrm{Ca}^{2+}$ channel regulation. Journal of molecular biology 355, 34-46 (2006). 
60. P. Beguin et al., Regulation of $\mathrm{Ca}^{2+}$ channel expression at the cell surface by the small G-protein kir/Gem. Nature 411, 701-706 (2001).

61. B. S. Finlin, S. M. Crump, J. Satin, D. A. Andres, Regulation of voltage-gated calcium channel activity by the Rem and Rad GTPases. P Natl Acad Sci USA 100, 1446914474 (2003).

62. C. T. Eggers, J. C. Schafer, J. R. Goldenring, S. S. Taylor, D-AKAP2 interacts with Rab4 and Rab11 through its RGS domains and regulates transferrin receptor recycling. J Biol Chem 284, 32869-32880 (2009).

63. S. A. Neumann et al., AKAP10 (I646V) functional polymorphism predicts heart rate and heart rate variability in apparently healthy, middle-aged European-Americans. Psychophysiology 46, 466-472 (2009).

64. R. E. Dixon et al., Graded $\mathrm{Ca}^{2+} /$ calmodulin-dependent coupling of voltage-gated Cav1.2 channels. Elife 4 (2015).

65. R. E. Dixon, C. Yuan, E. P. Cheng, M. F. Navedo, L. F. Santana, $\mathrm{Ca}^{2+}$ signaling amplification by oligomerization of L-type Cav1.2 channels. Proc Natl Acad Sci U S A 109, 1749-1754 (2012).

66. S. A. Tiscione et al., Disease-associated mutations in Niemann-Pick type $\mathrm{C} 1$ alter ER calcium signaling and neuronal plasticity. J Cell Biol 218, 4141-4156 (2019).

67. O. Vivas, S. A. Tiscione, R. E. Dixon, D. S. Ory, E. J. Dickson, Niemann-Pick Type C Disease Reveals a Link between Lysosomal Cholesterol and Ptdlns $(4,5) \mathrm{P}_{2}$ That Regulates Neuronal Excitability. Cell Rep 27, 2636-2648 e2634 (2019). 